\title{
WestVirginiaUniversity
}

THE RESEARCH REPOSITORY @ WVU

Graduate Theses, Dissertations, and Problem Reports

2011

\section{A Comparison of Methods for Evaluating Descriptive Analyses}

Ellen N. Brosh

West Virginia University

Follow this and additional works at: https://researchrepository.wvu.edu/etd

\section{Recommended Citation}

Brosh, Ellen N., "A Comparison of Methods for Evaluating Descriptive Analyses" (2011). Graduate Theses, Dissertations, and Problem Reports. 3308.

https://researchrepository.wvu.edu/etd/3308

This Thesis is protected by copyright and/or related rights. It has been brought to you by the The Research Repository @ WVU with permission from the rights-holder(s). You are free to use this Thesis in any way that is permitted by the copyright and related rights legislation that applies to your use. For other uses you must obtain permission from the rights-holder(s) directly, unless additional rights are indicated by a Creative Commons license in the record and/ or on the work itself. This Thesis has been accepted for inclusion in WVU Graduate Theses, Dissertations, and Problem Reports collection by an authorized administrator of The Research Repository @ WVU. For more information, please contact researchrepository@mail.wvu.edu. 
A Comparison of Methods for Evaluating Descriptive Analyses

\title{
Ellen N. Brosh
}

\author{
Thesis submitted to the \\ Eberly College of Arts and Sciences \\ at West Virginia University \\ in partial fulfillment of the requirements \\ for the degree of \\ Master of Science \\ in \\ Psychology \\ Claire St. Peter Pipkin, Ph.D., Chair \\ Tracy Morris, Ph.D. \\ Elizabeth Kyonka, Ph.D. \\ Department of Psychology
}

Morgantown, West Virginia

2011

Keywords: Descriptive Analysis, Functional Behavioral Assessment, Problem Behavior 


\begin{abstract}
A Comparison of Methods for Evaluating Descriptive Analyses
\end{abstract}

\title{
Ellen N. Brosh
}

Descriptive analyses, in which observers record behavior in the natural environment, are the most common procedure for completing functional behavioral assessments in schools. Because numerous studies have demonstrated that descriptive analyses do not consistently identify response-reinforcer relations, the frequent use of these assessments by educators is problematic. Attempts to improve the accuracy of descriptive analyses have focused on methods for analyzing descriptive assessment data. The current study compared three methods of analysis commonly cited in the behavior-analytic literature to determine whether these analyses produced similar results. Additionally, we included a treatment component to evaluate whether these methods of analysis produced effective interventions. For all participants, identical outcomes were obtained across at least two methods of descriptive analysis. For one of the participants, results from the descriptive analysis resulted in an effective intervention. 


\section{Acknowledgements}

I would like to thank Claire St. Peter Pipkin, Tracy Morris, and Elizabeth Kyonka for serving as members of my thesis committee and for their valuable contributions to the preparation of this manuscript. 
Table of Contents

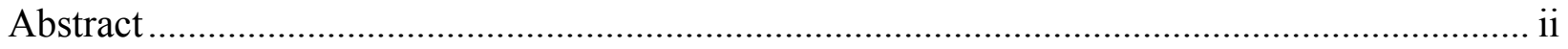

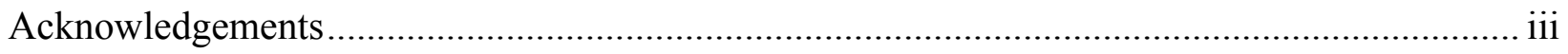

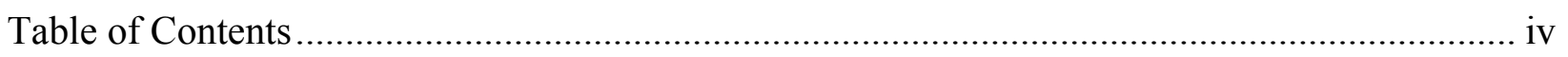

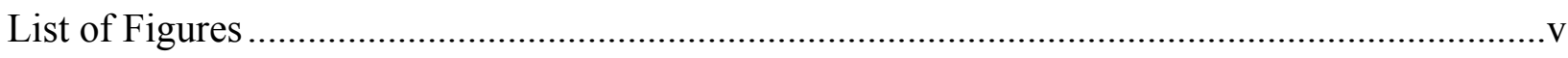

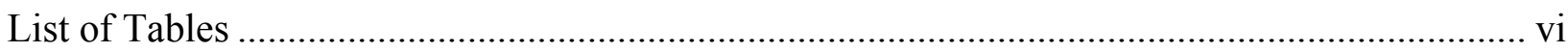

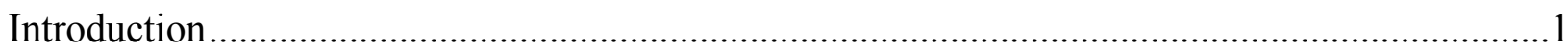

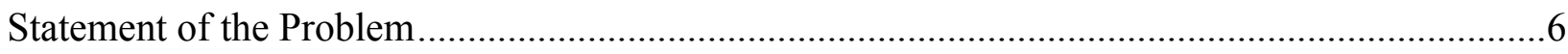

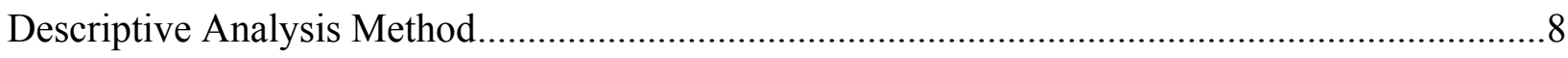

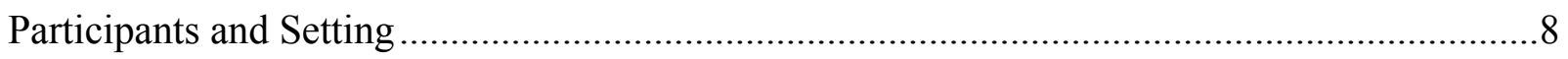

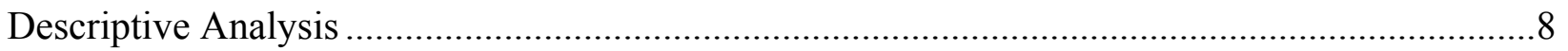

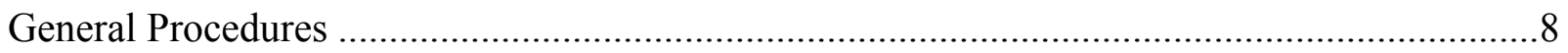

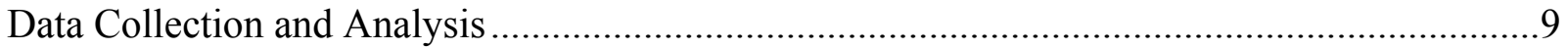

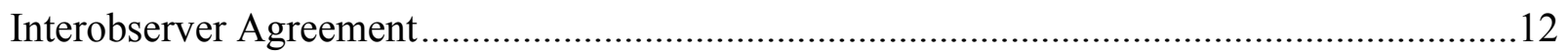

Probabilities of Behavior Given Event and Probabilities of Event Given Behavior. ................12

Conditional Probability Given Occurrence and Nonoccurrence of Problem Behavior ............15

Conditional Probabilities Versus Background Probabilities ...............................................17

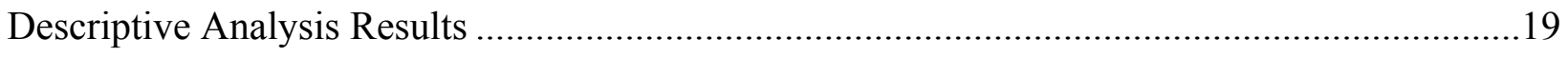

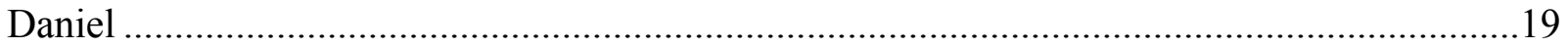

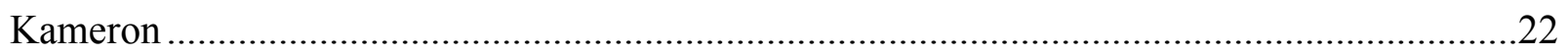

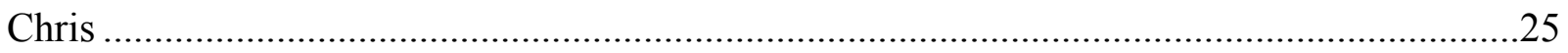

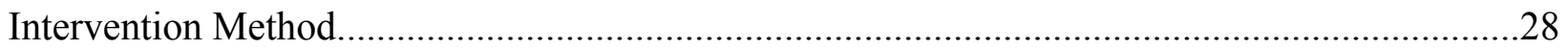

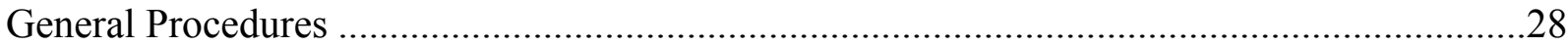

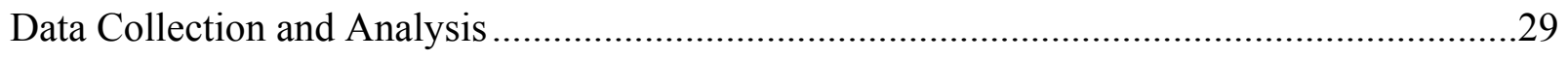

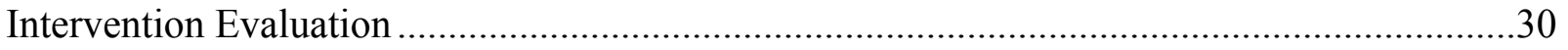

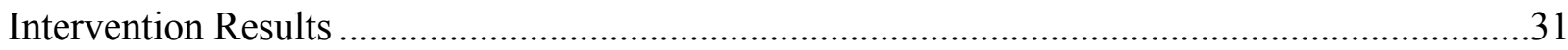

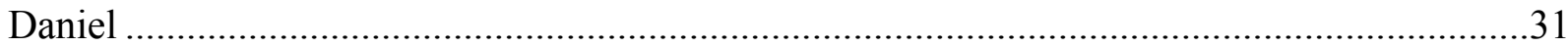

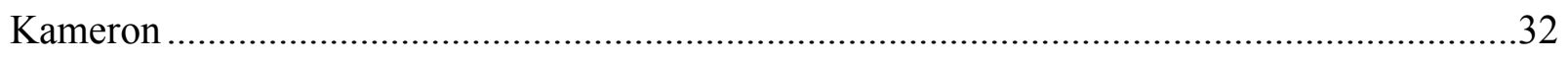

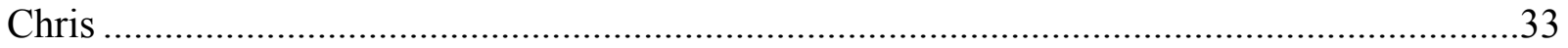

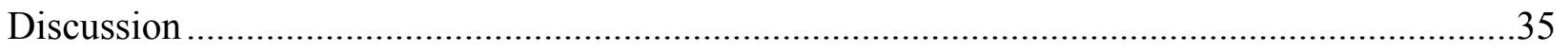

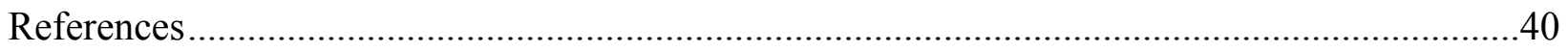




\section{List of Figures}

Figure 1. Graph of Daniel's descriptive analysis data analyzed according to the method described by Lerman and Iwata (1993).

Figure 2. Graph of Daniel's descriptive analysis data analyzed according to the methods of analysis described by Martens et al. (2008) and Vollmer et al. (2001). The top panel represents Daniel's data analyzed according to the method described by Martens et al. and the bottom panel represents Daniel's data analyzed according to the method described by Vollmer et al..47

Figure 3. Graph of Kameron's descriptive analysis data analyzed according to the method

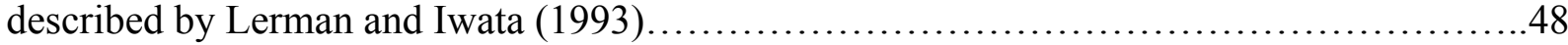

Figure 4. Graph of Kameron's descriptive analysis data analyzed according to the methods described by Martens et al. (2008) and Vollmer et al. (2001). The top panel represents Kameron's descriptive analysis data analyzed according to the method described by Martens et al and the bottom panel represents Kameron's data analyzed according to the method described by Vollmer et al.

Figure 5. Graph of Chris's descriptive analysis data analyzed according to the method described by Lerman and Iwata (1993). .50

Figure 6. Graph of Chris's descriptive analysis data analyzed according to the methods described by Martens et al. (2008) and Vollmer et al. (2001). The top panel represents Chris's data analyzed according to the method described by Martens et al. and the bottom panel represents Chris's data analyzed according to the method described by Vollmer et al..........51

Figure 7. Graph of treatment components for all participants. The top panel displays treatment results for Daniel and the middle panel displays treatment results for Kameron. 52

Figure 8. Graph of treatment results for Chris. The top panel displays treatment results from the attention and access intervention and the bottom panel displays treatment results from the break intervention 


\section{List of Tables}

Table 1. Probabilities calculated for each method of analysis ..................................................44

Table 2. A comparison of the putative reinforcers identified for each participant across the three

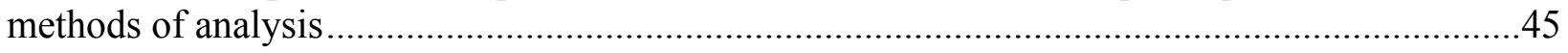




\section{Introduction}

The development of functional behavioral assessments (FBA) has substantially contributed to the field of behavior analysis. By identifying the environmental events maintaining behavior, FBAs have improved the efficacy and efficiency of behavioral interventions (Horner, 1994). Prior to FBAs, interventions relied on behavior modification techniques, which involved altering behavior without examining ongoing behavior-environment interactions (Mace, 1994). Although these interventions resulted in desirable effects (decreases in problem behavior and increases in appropriate behavior), the use of behavior modification techniques led to a dependence on the "default technologies of positive reinforcement and contingent aversive stimulation" (Mace, p. 386). Specifically, the effectiveness of these interventions relied on the use of strong punishers and reinforcers, rather than the identification and manipulation of the reinforcers maintaining the behavior. Functional behavior assessments created a technology for identifying relations between behavior and environmental events. Thus, interventions based on FBAs are designed to weaken existing response-reinforcer relations and lead to more robust treatments (Mace).

In 1997, the Individuals with Disabilities Education Act (IDEA) required educators to conduct FBAs for students engaging in problem behavior (Kern, Hilt, \& Gresham, 2004). The IDEA did not define or describe the components required for completing an FBA. Consequently, educators have a choice among a number of different FBA methodologies.

Broadly defined, FBAs involve a systematic process in which the factors that contribute to the occurrence and maintenance of behavior are identified (Sugai, Lewis-Palmer, \& HaganBurke, 2000). Functional analyses (FA), in which antecedents and consequents are systematically manipulated, remain the gold standard for identifying the function of behavior (Hanley, Iwata, \& McCord, 2003). Functional analyses are effective across a number of settings, 
populations, and behavior topographies. For example, functional analyses have been used to identify the reinforcers maintaining property destruction (Fisher, Lindauer, Alterson, \& Thompson, 1998), vocal tics (Carr, Taylor, Wallander, \& Reiss, 1996), bizarre vocalizations (Durand \& Crimmins, 1987), aggression (Pinkston, Reese, LeBlanc, \& Baer; 1973), and hair pulling (Miltenberger, Long, Rapp, Lumley, \& Elliot, 1998). Functional analyses may be superior to other forms of FBAs because they use experimental manipulation to identify functional relations, rather than naturalistic observations to identify correlational associations.

Functional analyses are not frequently used in school settings. Weber, Killu, Derby, and Barretto (2005) determined which FBA components were discussed most frequently in FBA materials provided to educators. Of the 41 states reviewed, 36 of the states' materials focused on direct observation and only 11 of the states' materials included experimental (functional analyses) manipulation.

The most common procedures used in schools to complete FBAs are descriptive analyses (DAs), which involve observations of the target behavior in the natural environment (Kern et al. 2004; Weber et al., 2005). During these observations, observers record instances of student and teacher behavior. Based on data collected during these observations, hypotheses are generated about environmental events maintaining the target behavior. Strong correlations between the target behavior and an environmental event indicate the event may be a reinforcer for the students' behavior (Lerman \& Iwata, 1993).

Because results obtained through descriptive analyses do not match results obtained through functional analyses, the frequent use of descriptive analyses by educators may be problematic. Thompson and Iwata (2007) compared the outcomes of descriptive analyses and functional analyses for 12 subjects and found that the two assessments produced similar results 
for only 3 of the subjects. Similarly, Hall (2005) compared the results of descriptive analyses and functional analyses of 4 subjects and found that descriptive analysis results matched the functional analysis results for only 1 subject. This disparity indicates that descriptive analyses may not consistently identify response-reinforcer relations.

A number of studies have improved the utility of descriptive analyses by pairing them with functional analyses. For example, Mace and Lalli (1991) combined descriptive analysis and functional analysis procedures to decrease one subject's aberrant speech. The descriptive analysis was first implemented to generate hypotheses concerning the function of the subject's problem behavior. The hypotheses generated from the descriptive analysis were tested in a functional analysis, and an intervention based on the results of the assessments was implemented. Although the intervention based on the results of the descriptive analysis and functional analysis effectively reduced the bizarre speech of the subject, combining descriptive analyses and functional analyses requires additional time and resources, which may make such combinations impractical in school settings.

Additional attempts to improve the utility of descriptive analyses have focused on methods for analyzing descriptive analysis data. The majority of these methods of analysis have involved the calculation of conditional probabilities of consequent events. Reliance only on conditional probabilities of consequent events however, often generated false hypotheses. For example, Lalli, Browder, Mace, and Brown (1993) calculated conditional probabilities of consequent events to identify the function of 3 students' problem behavior. Although the assessment identified a reinforcer for 2 of the 3 students, it generated multiple hypotheses for the remaining subject. Experimental manipulations confirmed only one of the hypotheses for this 
participant, indicating that an intervention based on both hypotheses may have resulted in an ineffective or unnecessarily complicated treatment.

Additional methods of analysis have involved calculations of probabilities of antecedent and consequent events. To date, three main analytic strategies have been reported in the literature (Martens, DiGennaro, Reed, Szczech, \& Rosenthal, 2008). These strategies are outlined below and the details of each procedure are described in detail in the methods section.

\section{Probabilities of Behavior Given Event and Probabilities of Event Given Behavior}

Lerman and Iwata (1993) calculated conditional probabilities of antecedent, consequent, and concurrent events. To demonstrate the utility of this method, Lerman and Iwata (1993) collected and analyzed descriptive analysis data for 6 subjects and compared results from the descriptive analyses to results from functional analyses. Results of the descriptive and functional analyses matched for only 1 of the 6 subjects. For 5 of the 6 subjects, results from the descriptive analysis suggested both a positive and negative reinforcement function (attention or escape), but did not discriminate between the two functions. Because no treatment data were presented, however, the utility of this method of analysis for intervention development cannot be verified.

\section{Conditional Probability Given Occurrence and Nonoccurrence of Problem Behavior}

Martens et al. (2008) hypothesized that relations between behavior and environmental events could be identified by calculating two conditional probabilities, the probability of a consequence given the behavior and the probability of a consequence given the absence of behavior. The relations between behavior and environmental events could be visually inspected by plotting these probabilities on a coordinate space. 
To demonstrate the utility of this method, Martens, Gertz, Werder, and Rymanowski (2010) collected and analyzed descriptive analysis data for 3 subjects and compared results from the descriptive analyses to results from functional analyses. Results from the descriptive analysis were compared to results from functional analysis test conditions that mimicked the natural environment, in that the participant's teacher, or a same-sex experimenter, implemented functional analysis test conditions. Martens and colleagues found that for 2 of the 3 subjects, outcomes from the descriptive analysis were consistent with results from the functional analysis. Because no treatment data were presented, however, the utility of this method of analysis for intervention development cannot be verified.

\section{Conditional Probabilities and Background Probabilities}

Vollmer, Borrero, Wright, Van Camp, and Lalli (2001) compared conditional probabilities of consequent events (the likelihood of the event given the occurrence of the behavior) and background probabilities of consequent events (the likelihood of the event independent of the behavior) for 11 subjects. Data were summed across observations and conditional and background probabilities were calculated for each of three consequent events (attention, access to materials, and escape from demands). The two probabilities were compared to identify positive, negative, and neutral contingencies between behavior and environmental events. For 3 subjects, positive contingencies, in which the conditional probability of an event was higher than the background probability of an event, were identified between behavior and at least one environmental event. Vollmer et al. concluded that events with positive contingencies may serve as reinforcers for the subject. For another 3 subjects, negative contingencies, in which the conditional probability of an event was lower than the background probability of an event, were identified between behavior and environmental events. For 3 additional subjects, neutral 
contingencies, in which the conditional probability of an event was the same as the background probability of an event, were identified between behavior and environmental events. The authors concluded that events with negative or neutral contingencies would not serve as reinforcers for the subject. The remaining participants' data were not provided. Vollmer et al. did not compare their results to results from a functional analysis or implement an intervention based on the results of the descriptive analysis, therefore, the utility of this method for generating effective interventions is unknown.

\section{Statement of the Problem}

The IDEA legislation requires FBAs for students who engage in problem behavior. Because the IDEA does not define or describe the components of an FBA, a number of different methodologies are used in schools. Although functional analyses are the most effective methodology for completing FBAs (Mace, 1994), such assessments are infrequently used in schools. Kern et al. (2004) found that naturalistic observations were the most common method for completing FBAs in schools for children with or at-risk for emotional behavioral disorders. In addition, Weber et al. (2005) found that the majority of FBA resources provided to educators focus on direct observation (descriptive analysis) rather than experimental manipulation of potential reinforcers.

The frequent use of descriptive analyses in schools may be problematic because such procedures often produce results that differ from experimental functional analyses (Hall, 2005; Thompson \& Iwata, 2007). This inconsistency indicates descriptive analyses may lead to ineffective interventions because appropriate response-reinforcer relations may not be identified. The utility of descriptive analyses in developing interventions, however, has been infrequently assessed. 
A number of studies have used a descriptive analysis to inform a subsequent functional analysis (e.g., Lalli, Browder, \& Mace, 1993; Mace \& Lalli, 1991). However, in school settings, where time and resources are limited, conducting both a descriptive analysis and functional analysis would often be impractical. Additional attempts at improving the accuracy of descriptive analyses have targeted the methods employed for analyzing descriptive analysis data. These methods have relied on an analysis of conditional probabilities of antecedent and consequent events for identifying environmental variables that may contribute to the target behavior. Three different methods for analyzing descriptive analysis are commonly reported in the literature (Martens et al., 2008).

The first method was introduced by Lerman and Iwata (1993) and includes a calculation of conditional probabilities of antecedent, consequent, and concurrent events. Lerman and Iwata did not include a treatment component, however, so it is unclear whether this method results in effective interventions. The second method of analysis, proposed by Martens et al. (2008), includes the calculation of two conditional probabilities, the probability of a consequence given the behavior and the probability of a consequence given the absence of the behavior. Similar to the study by Lerman and Iwata, Martens et al. also did not include a treatment component; therefore, the utility of this method for generating an effective intervention is unknown. The third method of analysis, developed by Vollmer et al. (2001), includes a comparison of conditional and background probabilities. Vollmer et al. also did not include a treatment component; therefore, it is not clear whether this method generates effective interventions.

The purpose of the current study was to extend the current literature on descriptive analyses in a number of ways. First, we compared the three methods of analysis to identify whether these analyses produced similar results. Second, we included a treatment component to 
evaluate whether the methods of analysis described by Lerman and Iwata (1993), Martens et al. (2008), and Vollmer et al. (2001) produce effective interventions.

\section{Descriptive Analysis Method}

\section{Participants and Setting}

Three elementary school students who received special education services participated. All participants engaged in problem behavior that disrupted school activities. Caregivers and teachers of each participant provided consent prior to participation in the study. Daniel was a 7year old male diagnosed with autism who engaged in aggression. Kameron was a 5-year old male diagnosed with pervasive developmental disorder who engaged in screaming. Chris was a 6-year-old male diagnosed with autism who engaged in disruptive and inappropriate vocal behaviors.

Data collection occurred in various rooms of the participant's school buildings. The exact location varied depending on the naturally occurring activities of the school day and the phase of the study. Data were collected by trained observers during school hours.

\section{Descriptive Analysis}

General procedures. Prior to beginning data collection, the experimenter received consent from the parents and the teachers, and explained to the teachers that she was observing student/teacher interactions. During data collection, the observer sat at least $3 \mathrm{~m}$ from the target student and remained as unobtrusive as possible. The observer ignored any attempts made by the student to interact.

Observers recorded target responses on laptop computers that had been programmed with a real-time data collection program (InstantData v1.1 for PC). This program allowed observers to record response frequency or duration. Each response was assigned a particular key on the 
computer keyboard. Responses that were discrete, including aggression, disruption, screaming, and inappropriate vocal behaviors, were recorded using frequency measures. For discrete responses, the observer pressed the corresponding key to record an occurrence. Responses that were continuous, including delivery of attention, access to materials, or breaks from demands, were recorded using duration measures. For continuous responses, the observer pressed the corresponding key at the onset of the response. This key remained active until the observer pressed the key again to indicate offset of the response. This observation system has been used in previous studies (e.g., St. Peter et al., 2005).

Observational data were collected for $4 \mathrm{hr}$ for each participant. Each observation lasted at least $10 \mathrm{~min}$, with the exact duration varying depending on the naturally occurring transitions in the school day. Data were collected on a real-time, second-by-second basis.

Data collection and analysis. Data were collected on student and teacher behavior. Topographies of targeted student behavior varied across participants. Aggression (Daniel) was defined as attempting to hit, kick, bite, head butt, or grab forcefully at appendages. Screaming (Kameron) was defined as vocalizations above conversation level. Disruption (Chris) was defined as throwing an item (not within $0.5 \mathrm{~m}$ of a person), banging on furniture or walls, tipping furniture, or ripping items. Inappropriate vocalizations (Chris) were defined as vocalizations above conversation level, whining, noncompliant statements (e.g., "No," "I don't want to"), or complaints about the activity (e.g., "This is stupid"). Data on these target behaviors were used to evaluate conditional probabilities of the behavior given various antecedent and subsequent events.

Data were also collected on teacher behavior. Measures were consistent across teachers and consisted of responses targeted in prior research, including delivery of attention, access to 
leisure items, and breaks, which were all scored as duration measures. Delivery of attention was defined as the teacher verbally or physically interacting with the student, including (but not limited to) reprimands, instructions, comfort statements, manual restraint, or hugs. Access to leisure items was defined as the availability of tangible items (e.g., food, computer) provided either by the teacher or accessed independently by the student (i.e., student takes item without permission), but did not include the availability of school items (e.g., worksheets, pencils, etc.). Breaks were defined as the absence of requests or demands by the teacher, and the student not complying with any requests or demands previously delivered by the teacher. Onset of breaks was scored after $3 \mathrm{~s}$ of no requests or demands by the teacher and the student not complying with any requests or demands previously delivered by the teacher. Offset of breaks occurred $3 \mathrm{~s}$ after requests or demands by the teacher or $3 \mathrm{~s}$ of the student complying with a request or demand previously delivered by the teacher. These definitions are consistent with prior research (e.g., Thompson \& Iwata, 2001).

Data collected on teacher behavior were used to calculate the probabilities of these events occurring before and after the targeted student behavior. To complete the methods of analysis described by Lerman and Iwata (1993) and Martens et al. (2008), antecedent teacher behavior was defined as those events (attention, access, break) occurring in the interval immediately prior to an interval containing the student's target behavior. For example, if the teacher provided the student with computer access and the student engaged in aggression in the following interval, access would be scored as an antecedent event. To complete the method of analysis described by Vollmer et al. (2001), antecedent teacher behavior was defined as those events (attention, access, break) occurring within the $10 \mathrm{~s}$ prior to the student's target behavior. For example, if the 
teacher provided the student with computer access and the student engaged in aggression within $10 \mathrm{~s}$, access would be scored as an antecedent event.

To complete the method of analysis described by Lerman and Iwata (1993) and Martens et al. (2008), subsequent events were defined as those events occurring in the interval following an interval containing the student's target behavior. For example, if a student engaged in aggression and was provided with a break in the following interval, break was scored as a subsequent event. To complete the method of analysis described by Vollmer et al. (2001), subsequent events were defined as those events occurring within $10 \mathrm{~s}$ following the target behavior. For example, if a student engaged in aggression and was provided with a break within $10 \mathrm{~s}$, break was scored as a subsequent event. Although some studies have scored events that occur within $5 \mathrm{~s}, 10 \mathrm{~s}, 15 \mathrm{~s}$, and $20 \mathrm{~s}$ as subsequent events, evaluation of these intervals has demonstrated that intervals longer than $10 \mathrm{~s}$ do not produce advantageous results for identifying reinforcers (Vollmer et al., 2001).

Data on teacher behavior were also used to infer the presence of three establishing operations (EOs) targeted in prior research: low attention, restricted access to materials, and demands. Establishing operations are events that may temporarily increase the value of a reinforcer (Michael, 1993). For example, during a period of low attention, the value of attention may increase. The EOs of low attention, restricted access to materials, and demands were assumed in the absence of attention, access to materials, and break from demands, respectively. These data were used to calculate conditional and background probabilities of events occurring within these EOs. The calculation was identical to that done by Vollmer et al. (2001) and will be explained in a subsequent paragraph. 
Interobserver agreement. Prior to collecting descriptive analysis data, potential observers were required to score videotapes of subjects similar to those participants in the current experiment. The potential observer's data were compared to the data of reliable observers. Potential observers were required to achieve interobserver agreement (IOA) scores (calculated as described below) of at least $90 \%$ on all responses during three consecutive sessions. Once potential observers met this criterion for videotapes of two subjects, they were considered trained to collect descriptive analysis data.

Interobserver agreement was calculated by dividing observations into 10 -s intervals. For each interval, the smaller number of responses recorded was divided by the larger number of responses recorded and multiplied by 100, yielding a percentage agreement. Percentage agreements were averaged across intervals to attain an IOA score for the entire session. Interobserver agreement was calculated for all responses. Interobserver data were collected during $64 \%, 33 \%$, and $31 \%$ of observations for Daniel, Kameron, and Chris, respectively. Interobserver agreement for Daniel was $95 \%$ for aggression (range, $88 \%$ to $100 \%$ ), $81 \%$ for attention delivery (range, $77 \%$ to $87 \%$ ), $88 \%$ for access to leisure items (range, $75 \%$ to $96 \%$ ), and $79 \%$ for break, (range, $70 \%$ to $96 \%$ ). Interobserver agreement for Kameron was $93 \%$ for screaming (range, $87 \%$ to97\%), 84\% for attention delivery (range, $77 \%-94 \%$ ), $94 \%$ for access to leisure items (range, $89 \%$ to $100 \%$ ), and $90 \%$ for break from demands (range, $73 \%$ to $99 \%$ ). Interobserver agreement for Chris was $100 \%$ for disruption, $94 \%$ for inappropriate vocalizations (range, $86 \%$ to $98 \%$ ), $86 \%$ for attention delivery, $99 \%$ for access to leisure items (range, $98 \%$ to $100 \%$ ), and $96 \%$ for break from demands (range, $92 \%$ to $100 \%$ ).

Probabilities of behavior given event and probabilities of event given behavior. The first method for analyzing the descriptive analysis data involved comparing conditional probabilities 
of antecedent events and subsequent events. The procedure was similar to the procedure described by Lerman and Iwata (1993); however, two modifications were made. Lerman and Iwata examined the conditional probabilities of antecedent, subsequent, and concurrent events. The authors concluded that conditional probabilities of concurrent events did not produce results beneficial for examining descriptive analysis data. Therefore, in the current study, only antecedent and subsequent conditional probabilities were compared. The second modification was a revision to the categories of antecedent and subsequent events. Lerman and Iwata combined attention and instructions into one variable, interaction, citing that this grouping did not produce any difference in data interpretation. Therefore, in the analysis by Lerman and Iwata, the probability of interaction was calculated by combining attention and instructions, and the probability of no interaction was calculated by combining the absence of attention and the absence of instructions. To allow for consistent comparisons between the three analyses used in the current study, we did not combine instructions and attention.

For each antecedent and consequent event, two conditional probabilities were calculated: the probability of the event given the behavior (intervals containing behavior and event divided by total number of intervals with behavior) and the probability of the behavior given the event (intervals containing event and behavior divided by total number of intervals with event). Table 1 lists the conditional probabilities that were calculated.

Prior to calculating probabilities, observation data were combined into 30-min observation blocks. For each 30-min observation block, two probabilities were calculated for each antecedent and consequent event. To calculate conditional probabilities of the event given the behavior, the number of 10 -s observation intervals containing the target behavior that were preceded by an observation interval containing a particular event (antecedent conditional 
probability) or followed by an observation interval containing a particular event (consequent conditional probability), were divided by the total number of observation intervals containing the behavior. For example, if the target behavior occurred during 10 observation intervals, and 8 of those occurrences were preceded by an interval containing attention, the antecedent conditional probability of attention would be 0.8 . If 6 of those occurrences were followed by an interval containing attention, the consequent conditional probability of attention would be 0.6 .

High proportions would indicate that events were more likely to precede or follow problem behavior than occur independent of problem behavior. Low proportions would indicate that events were more likely to be provided independent of problem behavior than preceding or following problem behavior. This would suggest that these events were not likely to evoke or be maintaining problem behavior. As with Lerman and Iwata's original analysis, EOs were not taken into consideration when calculating conditional probabilities.

To calculate conditional probabilities of the behavior given the event, the number of 10 -s observation intervals containing a particular event that were preceded by an interval containing the behavior (antecedent conditional probability) or followed by an interval containing the behavior (consequent conditional probability), were divided by the total number of observation intervals with the event. For example, if attention occurred during 10 observation intervals, and 4 of those occurrences were preceded by an interval containing the behavior, the antecedent conditional probability would be 0.4 . If 7 of those occurrences were followed by an interval containing the behavior, the consequent conditional probability would be 0.7 . High proportions would indicate that problem behavior was more likely to precede or follow events than to occur in the absence of these events. Low proportions would indicate that problem behavior was more likely to occur in the absence of these events than preceding or following these events. 
For each participant, conditional probabilities for all antecedent and subsequent events were plotted on four line graphs (see Figure 1 for example), with each graph depicting a different probability. The top left panel of each figure shows the proportion of event intervals (low attention, restricted access to materials, and demands) occurring prior to problem behavior intervals. The top right panel displays the proportion of problem behavior intervals that occurred following event intervals. These calculations provide information about antecedent events that may evoke problem behavior. For example, behavior frequently preceded by low attention suggests that periods of low attention may evoke problem behavior.

The bottom left panel of each figure displays the proportion of event intervals (attention, access to materials, and break from demands) that occurred after problem behavior intervals. The bottom right panel displays the proportion of problem behavior intervals that were followed by event intervals. These calculations provide information about consequent events that may serve as reinforcers for problem behavior. For example, behavior frequently followed by attention suggests that attention may function as a reinforcer for problem behavior. Graphs were visually inspected to identify events that may function as reinforcers.

Conditional probability given occurrence and nonoccurrence of problem behavior. The second method of analysis examined conditional probabilities using a strategy known as a contingency space analysis. The procedure was identical to that described by Martens et al. (2008). For each observation, two conditional probabilities were calculated for each event: the probability of a consequence given behavior and the probability of a consequence given the absence of behavior. The bottom section of Table 1 displays the conditional probabilities that were calculated for the contingency space analysis. 
Prior to calculating probabilities, observation data were combined into 30-min observation blocks. Each 30-min observation block was then further divided into continuous 10s observation intervals. To calculate the conditional probability of a consequence given the behavior, the number of observation intervals containing the target behavior that were followed by an interval containing a particular event were divided by the total number of observation intervals containing the behavior. To calculate the probability of a consequence given the absence of behavior, the number of observation intervals containing an event that were not followed by an interval containing the target behavior were divided by the total number of intervals containing no target behavior. Martens et al. (2008) did not specify the level of aggregation for the probabilities (i.e. for each observation session or by summing the data across observation sessions). In the current study, conditional probabilities were calculated for each observation session, as well as by summing across observation sessions.

Probabilities for each observation block were graphed on a coordinate space (see top panel of Figure 2 for example) to identify contingencies between behavior and environmental events. Data averaged across all observations (left panel) and session-by-session data (right panel) were graphed. For both graphs, the x-axis depicts the probability of an event given the absence of problem behavior, and the y-axis depicts the probability of an event given the occurrence of problem behavior, with each type of event (attention, access, and break) denoted by a different symbol. Points falling on the diagonal line dividing the plane indicate situations in which a consequence is just as likely to occur given the occurrence or non-occurrence of the behavior. Points falling above the line represent consequent events that are more likely to occur given the target behavior; these are considered to be potential reinforcers. Points falling below the line represent events that are more likely to occur given the absence of the target behavior. 
Graphs were visually inspected to identify possible social reinforcers for problem behavior. On the graph displaying session-by-session data, those events with at least $50 \%$ of data points falling above the line were hypothesized as reinforcers for problem behavior. If more than $50 \%$ of data points for all events fall below the line, an automatic reinforcement function would be suggested. On the graph displaying data averaged across all sessions, those events with the single data point falling above the line were hypothesized as reinforcers for problem behavior.

Conditional probabilities versus background probabilities. The third method of analysis compared conditional probability values and background probability values within EOs. The procedure was similar to that described by Vollmer et al. (2001). Conditional probabilities identify the likelihood of an event given the occurrence of student target behavior. For example, the conditional probability of attention for aggressive behavior identifies the likelihood that attention would follow aggression. Background probabilities identify the probability of an event occurring at randomly selected points in time. For example, the background probability of attention identifies the likelihood of attention occurring during randomly selected seconds of an observation.

Vollmer et al. (2001) calculated event probabilities using two methods, a binary calculation and a proportion of seconds calculation. Because the proportion of seconds calculation did not produce results beneficial for analyzing the descriptive analysis data above those provided by the binary method alone, only the binary method was used in the current study. Binary calculations were computed using methods identical to those described by Vollmer and colleagues. Events (attention, access, break) were analyzed as either occurring (yes) or not (no) occurring following instances of target behavior (conditional probability) or 
during random seconds (background probabilities). Data were summed across observation sessions to generate a single conditional and background probability for each event.

The method described by Vollmer and colleagues (2001) is the only method that accounts for both antecedent and consequent events. Antecedent events are included because only those instances of behavior that occurred within an antecedent establishing operation (EO) are included in the analysis. Before calculating probabilities, seconds containing the EOs of low attention, restricted access to materials, and demands were identified. The total instances of problem behavior that occurred during each EO were calculated and summed across observation sessions. Conditional and background probabilities for each consequent event were computed within these EOs. Table 1 lists the formulas for conditional and background probabilities, as computed by Vollmer et al.

Data were summed across observation sessions to generate a single conditional and background probability for each event. Conditional probabilities of each consequent event were computed by dividing the number of instances of problem behavior during an EO (e.g., the absence of attention) that were followed within $10 \mathrm{~s}$ by a particular event (e.g., attention) by the total number of instances of problem behavior within the EO. For example, if 8 out of 10 occurrences of the target behavior within the EO of low attention were followed by attention within $10 \mathrm{~s}$, the conditional probability of attention would be 0.8 .

To calculate background probabilities, 50 seconds of each EO were randomly selected from all seconds in which the EO was in place. Because these seconds were randomly selected, it was possible for behavior to co-occur with the randomly selected seconds. If more than 50 occurrences of the target behavior occur during a particular EO, the number of seconds selected equaled the number of instances of the target behavior. Randomly selected seconds that were 
followed by an event within $10 \mathrm{~s}$ were divided by the total number of seconds selected to determine the background probability. For example, if 25 of the 50 selected seconds were followed by attention within $10 \mathrm{~s}$, the background probability of attention would be 0.5 .

Conditional probabilities and background probabilities were depicted on bar graphs (see bottom panel of Figure 2 for example). The x-axis shows events, and the y-axis shows probability value. Black bars represent conditional probabilities and gray bars represent background probabilities.

Graphs were visually inspected to identify positive, negative, and neutral contingencies between events and problem behavior (Vollmer et al., 2001). A positive contingency is one in which the conditional probability of a consequent event is higher than the background probability of the event. A positive contingency would suggest that the event may contribute to the maintenance of behavior. A negative contingency is one in which the conditional probability of the consequent event is lower than the background probability. Such contingencies suggest a relation in which the event is less likely to serve as a reinforcer for the problem behavior. A neutral contingency is one in which the conditional probability of the consequent event is identical to the background probability of the event. Such contingencies suggest no relation between the event and the behavior.

\section{Descriptive Analysis Results}

Daniel. Figures 1 and 2 depict data from Daniel's descriptive analysis. Figure 1 displays data analyzed according to the method of analysis described by Lerman and Iwata (1993). The top left panel shows that overall, low attention, restricted access to materials, and demands rarely occurred antecedent to problem behavior; these events did not reliably evoke problem behavior. The average proportion of intervals containing low attention, restricted access to materials, and 
demands that preceded problem behavior were $0.01,0.14$, and 0.14 , respectively. Intervals of restricted access and demands were slightly more likely to precede problem behavior than intervals of low attention. These results suggest that Daniel's problem behavior was more likely to be evoked by periods of restricted access to materials and periods of demands.

The top right panel of Figure 1 shows that when problem behavior did occur, it was more likely to be preceded by intervals of demands and restricted access to materials than by intervals of low attention. The average proportion of problem behavior intervals that were preceded by restricted access to material intervals and demand intervals were 0.51 , and 0.63 , respectively. The average proportion of problem behavior intervals that were preceded by low attention intervals was 0.02 . Similar to the top right panel, these data suggest that periods of restricted access and periods of demands may evoke problem behavior.

The bottom left panel of Figure 1 shows that low proportions of attention, access, and break intervals occurred consequent to problem behavior intervals. Thus, these events were more likely to occur following intervals without problem behavior than following intervals with problem behavior. The average proportions of attention, access to materials, and break from demand intervals that followed problem behavior were approximately equal, with average proportions of $0.08,0.05$, and 0.06 , respectively.

The bottom right panel of Figure 1 shows that when problem behavior did occur, it was generally more likely to be followed by periods of attention and access to materials than to be followed by periods of escape, although the data are highly variable. During observations 2, 4, 5 , and 6 , every interval with problem behavior was followed by attention. During observations 1,2 , and 3 , every interval with problem behavior was followed by an interval with access to leisure materials. The average proportions of problem behavior intervals that were followed by 
attention, access to materials, and break from demands were $0.72,0.68$, and 0.44 , respectively. These data suggest that attention and access to materials may serve as reinforcers for Daniel's problem behavior. Taken together, the four panels of Figure 1 suggest that Daniel's problem behavior may be maintained by any of the three included social reinforcers (attention, access to materials, and break from demands).

The top panel of Figure 2 displays Daniel's data analyzed according to the method described by Martens et al. (2008). The upper-left panel displays Daniel's data averaged across all observations. This analysis identified that attention may serve as a reinforcer for Daniel's problem behavior. The average probability of attention following the occurrence of problem behavior $(\mathrm{x}=0.73)$ was higher than the average probability of attention following the absence of problem behavior $(\mathrm{x}=0.68)$. This analysis identified that access to materials and break from demands may not serve as a reinforcer for problem behavior. The average probabilities of access to materials $(\mathrm{x}=0.68)$ and break from demands $(\mathrm{x}=0.43)$ occurring following problem behavior were lower than the average probabilities of access to materials $(x=0.76)$ and break from demands $(\mathrm{x}=0.61)$ following the absence of problem behavior.

The right panel of Figure 2 displays Daniel's data analyzed on an observation-byobservation basis. For $71 \%$ of observations, the probability of attention following the occurrence of problem behavior was higher than the probability of attention following the absence of problem behavior. For $57 \%$ and $86 \%$ of observations, the probabilities of access to materials and break from demands following the occurrence of problem behavior were lower than the probabilities of these events following the absence of problem behavior. Results, therefore, suggest attention as a reinforcer for Daniel's problem behavior. 
The bottom panel of Figure 2 displays Daniel's descriptive analysis data analyzed according to the method described by Vollmer et al. (2001). Results from this analysis were similar to results from the Martens et al. (2008) analysis. A positive contingency was identified between attention and problem behavior. The conditional probability of attention following problem behavior (1.00; this differs from the Martens et al. conditional probability because establishing operations were considered in the Vollmer et al. analysis, but not the Martens et al. analysis) was higher than the background probability of attention (0.21). Negative contingencies were identified between access to materials and problem behavior, and break from demands and problem behavior. The conditional probabilities of these events were lower than the background probabilities of these events. Conditional probabilities of access to materials and break from demands were 0 and 0.21 , respectively. Background probabilities of access to materials and break from demands were 0.21 and 0.26 , respectively. Thus, analyses described by Vollmer et al. and Martens et al. suggested that attention may contribute to the maintenance of Daniel's problem behavior.

Table 2 summarizes results from the three methods of analysis for Daniel. The method of analysis described by Lerman and Iwata (1993) identified attention, access to materials, and break from demands as reinforcers for Daniel's problem behavior. The method of analysis described by Martens et al. (2008) and Vollmer et al. (2001) identified attention as a reinforcer for problem behavior. Because all three events were identified as possible reinforcers by the analyses, all were included in the intervention evaluation.

Kameron. Figures 3 and 4 depict data from Kameron's descriptive analysis. Figure 3 depicts Kameron's data analyzed according to the method of analysis described by Lerman and Iwata (1993). The top left panel of Figure 3 shows that, overall, relatively low proportions of 
intervals containing periods of low attention $(x=0.14)$, restricted access to materials $(x=0.12)$, and demands $(\mathrm{x}=0.13)$ occurred antecedent to problem behavior. The probabilities of these events preceding problem behavior were approximately equal, suggesting that these antecedents are equally likely to evoke problem behavior.

The top right panel of Figure 3 shows that when problem behavior did occur, it was most often preceded by periods of low attention. The average proportion of problem behavior preceded by low attention was 0.67 . Periods of restricted access to materials and demands also reliably preceded problem behavior, with proportions of 0.34 and 0.43 , respectively. These data suggest that Kameron's problem behavior is most likely to be evoked by periods of low attention, but that periods of restricted access to materials and periods of demands may also evoke problem behavior.

The bottom left panel of Figure 3 shows that low proportions of intervals containing attention, access to materials, and break from demands occurred consequent to problem behavior. Intervals containing these events were more likely to occur after intervals without problem behavior than after intervals with problem behavior. Proportions of intervals of attention, access to materials, and break from demands that occurred following problem behavior were approximately equal. The average proportions of attention, access to materials, and break from demands that followed problem behavior intervals were $0.13,0.16$, and 0.14 , respectively. The bottom right panel of Figure 3 shows that when problem behavior did occur, high proportions were followed by access to materials and break from demands, with average proportions of 0.77 and 0.53 , respectively. These data suggest that access to materials and break from demands may function as reinforcers for Kameron's problem behavior. Although the average proportion of problem behavior intervals followed by attention intervals $(\mathrm{x}=0.29)$ was 
slightly less than proportions of access to materials and demands, during the final four observations, the proportion of problem behavior intervals followed by attention increased, suggesting attention may function as a reinforcer as well.

The top panel of Figure 4 displays Kameron's data analyzed according to the method of analysis described by Martens et al. (2008). The top left panel displays Kameron's data averaged across all observations. This analysis identified attention, access to materials, and break from demands as putative reinforcers for Kameron's problem behavior, as the probability of these events following the occurrence of problem behavior was higher than the probability of these events following the absence of problem behavior. The average conditional probabilities of attention, access to materials, and break from demands following problem behavior were 0.29, $0.77,0.59$, and the average conditional probabilities of these events following the absence of problem behavior were $0.28,0.63$, and 0.37 , respectively. The top right panel displays Kameron's data analyzed on an observation-by-observation basis. For 57\%, 57\%, and 86\% of observations, the probabilities of attention, access to materials, and break from demands following the occurrence of problem behavior was higher than the probability of these events following the absence of problem behavior, respectively. Results from this analysis suggest attention, access to materials, and break from demands as potential reinforcers for Kameron's problem behavior.

The bottom panel of Figure 4 displays Kameron's data analyzed according to the method of analysis described by Vollmer et al. (2001). This analysis identified a positive contingency between attention and problem behavior. The conditional probability of attention following problem behavior $(0.25)$ was higher than the background probability of attention $(0.20)$. Negative contingencies were identified between access to materials and problem behavior and 
break from demands and problem behavior. The conditional probabilities of access to materials and break from demands were 0.03 and 0.05 , respectively. The background probabilities of access to materials and break from demands were 0.15 and 0.09 , respectively. Thus, results from this analysis suggest attention as a reinforcer for Kameron's problem behavior.

Table 2 summarizes results from the three methods of analysis for Kameron. The methods of analysis described by Lerman and Iwata (1993) and Martens et al. (2008) identified attention, access to materials, and break from demands as reinforcers for Daniel's problem behavior. The method of analysis described by Vollmer et al. (2001) identified attention as a reinforcer for problem behavior. Because all three events were identified as possible reinforcers by the analyses, all were included in the intervention evaluation.

Chris. Figures 5 and 6 depict the results from Chris's descriptive analysis. Figure 5 displays Chris's data analyzed according to the method of analysis described by Lerman and Iwata (1993). The top left panel shows that low proportions of intervals containing periods of low attention, restricted access to materials, and demands occurred antecedent to problem behavior. On average, however, periods of restricted access to materials $(x=0.13)$ and demands $(\mathrm{x}=0.16)$ were slightly more likely to precede problem behavior than intervals of low attention $(\mathrm{x}=0.06)$. These data suggest periods of restricted access and periods of demands may evoke problem behavior, although trends in the data suggest that these relations may have been weak or transient.

The top right panel shows that when problem behavior did occur, the most common antecedent was low attention. The average proportion of problem behavior intervals preceded by low attention intervals was 0.60 . The average proportions of problem behavior intervals 
preceded by restricted access to materials and demand intervals were 0.22 and 0.23 , respectively. Thus, this panel suggests attention as a reinforcer for Chris's problem behavior.

The bottom left panel of Figure 5 shows that low proportions of intervals containing attention, access to materials, and breaks from demands occurred consequent to problem behavior. Thus, intervals of attention, access to materials, and breaks from demands were more likely to follow intervals without problem behavior than follow intervals with problem behavior. The average proportion of attention intervals following problem behavior $(\mathrm{x}=0.15)$ was slightly higher than the average proportion of access to material intervals $(x=0.06)$ or break intervals $(\mathrm{x}$ $=0.08)$ that followed problem behavior. These data suggest attention may serve as a reinforcer for Chris's problem behavior.

The bottom right panel of Figure 5 shows that when problem behavior did occur, high proportions were followed by break from demands $(\mathrm{x}=0.86)$, and moderate proportions were followed by attention $(x=0.50)$ and access to materials $(x=0.63)$. These results suggest attention, access to materials, and breaks from demands may serve as reinforcers for Chris's problem behavior.

The upper panel of Figure 6 displays Chris's data analyzed according to the method of analysis described by Martens et al. (2008). The upper-left panel displays Chris's data averaged across all observations sessions. This analysis identified attention as a possible reinforcer for Chris's problem behavior. The average probability of attention following the occurrence of problem behavior $(\mathrm{x}=0.50)$ was higher than the average probability of attention following the absence of problem behavior $(\mathrm{x}=0.28)$. This analysis identified that access to materials and break from demands may not serve as reinforcers for Chris's problem behavior. The average conditional probabilities of access to materials $(\mathrm{x}=0.63)$ and break from demands $(\mathrm{x}=0.86)$ 
following the occurrence of problem behavior were lower than the average conditional probabilities of access to materials $(\mathrm{x}=0.94)$ and break from demands $(\mathrm{x}=0.93)$ following the absence of problem behavior.

The right panel displays Chris's data on an observation-by-observation basis. For $86 \%$ of observations, the probability of attention following the occurrence of problem behavior was higher than the probability of attention following the absence of problem behavior. In addition, only $14 \%$ of access data points and $29 \%$ of break from demand data points fell above the diagonal line, indicating that during these sessions, the probability of these events following the occurrence of problem behavior was lower than the probability of these events following the absence of problem behavior. Thus, results from this analysis suggest attention as a reinforcer for Chris's problem behavior.

The bottom panel of Figure 6 displays Chris's data analyzed according to the method of analysis described by Vollmer et al. (2001). This analysis identified positive contingencies for all events, suggesting that attention, access to materials, and breaks from demands may serve as reinforcers for Chris's problem behavior. The conditional probabilities of attention (0.48), access to materials (0.21), and break from demands (0.47) were higher than the background probabilities these events. Background probabilities of attention, access to materials, and break from demands were $0.23,0.05$, and 0.31 , respectively.

Table 2 summarizes results from the three methods of analysis for Chris. The methods of analysis described by Lerman and Iwata (1993) and Vollmer et al. (2001) identified attention, access to materials and break from demands as reinforcers for Chris's problem behavior. The method of analysis described by Martens et al. (2008) identified attention as a reinforcer for 
problem behavior. Similar to the previous participants, because all three events were identified as possible reinforcers, all were included in the intervention evaluation.

\section{Intervention Method}

The purpose of the intervention evaluation was to evaluate the extent to which the reinforcers identified by the descriptive analyses resulted in effective interventions. Variables identified in the analyses as putative reinforcers were evaluated in a reversal design or a reversal design with an embedded multielement (see results section for details about the design). For example, if results from the descriptive analysis indicated that attention may serve as a reinforcer for problem behavior, attention was evaluated as a reinforcer in the intervention. Behavior change during the intervention determined whether the methods of analyses identified functional reinforcers for the participant's problem behavior.

Putative reinforcers were manipulated across conditions. The number of putative reinforcers identified by the analyses dictated the number of baseline conditions. For example, if attention and access were identified as putative reinforcers in the descriptive analysis, the evaluation included two baseline conditions: attention and access. If only one variable was identified by the descriptive analysis, then only one baseline condition was evaluated.

General procedures. Trained graduate students served as therapists. Sessions occurred in a relatively barren room of the student's school and were $5 \mathrm{~min}$ in duration. The therapist, the student, and data collectors were present during sessions. Phases consisted of a minimum of 3 sessions and a maximum of 10 sessions. The exact number of sessions per phase was determined by visual inspection of the data. When rates of problem behavior were stable or changing in the expected direction for three consecutive sessions of a baseline phase, the treatment phase began.

For example, if rates of problem behavior were increasing after three sessions during the baseline 
attention phase, the treatment attention phase was implemented. For evaluations conducted in a multielement design, the order of sessions within a phase was selected randomly without replacement.

Response rate was calculated for student responses by dividing the total number of occurrences of the behavior by the number of minutes per session. For each session, response rate was plotted on a line graph (see Figure 7 for example). Graphs were visually inspected to determine if the variables identified served as reinforcers.

Data collection and analysis. Data collection during sessions was similar to the methods used during the descriptive analysis. Data were collected on student problem behavior. The topography of the problem behavior was identical to the one during the descriptive analysis. Two independent observers collected data for $30 \%, 28 \%$, and $40 \%$, of the sessions for Daniel, Kameron, and Chris, respectively, and IOA was calculated as previously described. Interobserver agreement for Daniel was 100\% for aggression, 93\% for attention delivery (range, $82 \%$ to $99 \%$ ), $99 \%$ for access to leisure items (range, $97 \%$ to $100 \%$ ), and $99 \%$ for breaks from demand (range, $93 \%$ to $100 \%$ ). Interobserver agreement for Kameron was $96 \%$ for screaming (range, $74 \%$ to $100 \%$ ), $91 \%$ for attention delivery (range, $72 \%$ to $100 \%$ ), $99 \%$ for access to leisure items (range, $96 \%$ to $100 \%$ ), and $96 \%$ for breaks from demand (range, $86 \%$ to $99 \%$ ). Interobserver agreement for Chris was $98 \%$ for disruption (range, $92 \%$ to $100 \%$ ), $95 \%$ for inappropriate vocalizations (range, $82 \%$ to $100 \%$ ), $92 \%$ for attention delivery (range, $75 \%$ to $99 \%$ ), $98 \%$ for access to leisure items (range, $76 \%$ to $100 \%$ ), and $93 \%$ for breaks from demand (range, $86 \%$ to $100 \%$ ).

Observers also scored therapist responses. Data on therapist behavior was collected to calculate procedural integrity (the degree to which the therapist is implementing the intervention as specified). To calculate procedural integrity, a therapist response was scored as correct when 
the designated procedure was implemented within $2 \mathrm{~s}$ of the target behavior, or withheld in the absence of the target behavior. The total number of correct responses was divided by the total number of response opportunities, and reported as a percentage. Procedural integrity data were collected during $80 \%, 30 \%$, and 30\%, of sessions for Daniel, Kameron, and Chris, respectively. Procedural integrity was $100 \%, 100 \%$, and $98 \%$ (range, $75 \%$ to $100 \%$ ) during treatment sessions for Daniel, Kameron, and Chris, respectively.

Intervention evaluation. During baseline sessions, putative reinforcers were provided for $30 \mathrm{~s}$ following each instance of problem behavior. During the attention condition, the therapist withheld attention following instances of appropriate behavior and provided $30 \mathrm{~s}$ of attention each time the child engaged in problem behavior. Attention consisted of verbal statements delivered by the therapist in a neutral tone. During the access condition, the therapist restricted access to desired items and provided the student with $30 \mathrm{~s}$ of access to a desired item each time the child engaged in problem behavior. The desired item was an item that the student manipulated during the descriptive analysis and was described as preferred by the teacher. During the break condition, the therapist delivered demands using a three-step prompting sequence. The initial prompt consisted of a verbal statement requesting the student to complete a task, such as "Point to the circle." If the student did not respond within $10 \mathrm{~s}$, the therapist modeled the appropriate response, while repeating the verbal request. If the student did not comply within $10 \mathrm{~s}$ of the second prompt, the therapist physically guided the student to complete the task. If the student complied with a demand, the therapist delivered praise, consisting of a brief verbal statement, and then delivered the next task. Each time the student engaged in problem behavior, the therapist provided the student with a 30-s break. During the break, the 
therapist moved at least $1 \mathrm{~m}$ away from the child, discontinued the prompting sequence, and refrained from delivering additional demands or attention to the child.

If problem behavior did not occur during baseline, it suggested that the variable identified by the descriptive analysis was not a reinforcer for the behavior in the therapy context. If problem behavior did occur during baseline, those conditions in which the problem behavior occurred at rates greater than zero for three consecutive sessions were included in a treatment phase. Treatment sessions were identical to those in the baseline, except that putative reinforcers were withheld following instances of problem behavior and provided each time the child engaged in appropriate behavior. Following completion of the treatment, baseline and treatment phases were repeated to demonstrate experimental control in a reversal design.

\section{Intervention Results}

Daniel. The top panel of Figure 7 displays Daniel's data from the intervention evaluation. Daniel's intervention evaluation was conducted using a multielement design. Open circles depict problem behavior during attention baseline conditions, closed circles depict problem behavior during access baseline conditions, and closed triangles depict problem behavior during break baseline conditions. The criterion for moving from baseline sessions to treatment sessions was elevated rates of problem behavior during 3 consecutive sessions of a condition. Daniel did not meet the criterion for moving from baseline to treatment sessions for any condition. Daniel engaged in problem behavior during two attention sessions (sessions 5 and 8), one access session (session 27), and three nonconsecutive break sessions (sessions 2, 10, and 15). Because Daniel did not meet the criterion for moving to treatment sessions, his treatment evaluation was terminated after completing 10 sessions per condition. Daniel's data suggest that the events 
identified by the three analyses were not reinforcers for Daniel's problem behavior in the treatment context.

Kameron. The middle panel of Figure 7 displays Kameron's data from the intervention evaluation. Kameron's intervention evaluation was conducted using a reversal design. A reversal design was used with Kameron because previous evaluations with Kameron (a functional analysis conducted before this study) indicated that carry-over effects were problematic in a multi-element design. Closed circles depict behavior during attention conditions, open circles depict behavior during access conditions, and closed triangles depict behavior during break conditions.

Kameron never engaged in problem behavior during attention and access baseline conditions. These results indicated that exposure to short periods (sessions were $5 \mathrm{~min}$ in duration) of low attention and restricted access to materials were not sufficient for evoking problem behavior. Kameron engaged in elevated rates of problem behavior during the final 3 sessions of the break baseline condition. Across these sessions, Kameron engaged in problem behavior at an average rate of 2.2 instances of problem behavior per min. When the break-based treatment was introduced, rates of problem behavior immediately decreased. Kameron did not engage in problem behavior during the first five sessions of this condition. Bursts of problem behavior occurred during sessions 36 and 40 , but decreased in the final 3 sessions of the condition. When the break baseline was again implemented, Kameron's behavior did not return to baseline levels. Problem behavior remained suppressed across 10 consecutive sessions of the second break baseline. Kameron's behavior did not meet criterion for returning to the treatment condition after 10 break baseline sessions; therefore, the intervention evaluation for Kameron was terminated. Because Kameron's behavior did not return to baseline levels during the second 
exposure to baseline, it seems unlikely that break from demands was a reinforcer for Kameron's problem behavior.

Chris. Figure 8 displays Chris's data from the intervention evaluation for attention, access, and break conditions (to facilitate visual inspection of the data, Chris's data from the intervention evaluation were separated into two graphs). Chris's intervention evaluation was conducted using a reversal design. A reversal design was used with Chris because previous evaluations with Chris suggested that carry-over effects may be problematic in a multi-element design. The top panel of Figure 8 displays Chris's data from the intervention evaluation for attention and access conditions. Closed circles depict problem behavior during attention conditions and open circles depict problem behavior during access conditions. Chris did not meet the criterion for moving from baseline to treatment sessions for the attention condition. Chris engaged in problem behavior during three nonconsecutive attention baseline sessions (sessions 1, 7, and 9). These results indicate that exposure to short periods (sessions were $5 \mathrm{~min}$ in duration) of low attention were not sufficient for evoking problem behavior. Because Chris did not meet the criterion for moving to treatment sessions, his attention evaluation was terminated after completing 10 baseline sessions. These data suggest that attention was not a reinforcer for Chris's problem behavior in the treatment context.

Chris engaged in elevated rates of problem behavior during the access baseline condition. Across these sessions, Chris engaged in problem behavior at an average rate of 2.3 instances of problem behavior per min. When the access treatment was introduced, rates of problem behavior increased above baseline rates during the second session of this condition (session 16). Following this session, however, Chris's rates of problem behavior decreased. In the final three sessions of this condition, Chris engaged in 0.13 instances of problem behavior per min. When 
the access baseline was again implemented, Chris's rate of problem behavior returned to levels obtained in the previous baseline phase. During this baseline phase, Chris engaged in problem behavior at an average rate of 2.0 instances per min. When the access treatment was again implemented, Chris's rate of problem behavior decreased. In the three sessions of this phase, Chris engaged in problem behavior at an average rate of 0.07 instances per min. These data indicate that access to leisure items served as a reinforcer for Chris's problem behavior, and that providing leisure items contingent on appropriate behavior resulted in clinically significant treatment effects.

The bottom panel of Figure 8 displays Chris's results from the intervention evaluation for the break condition. Chris engaged in elevated rates of problem behavior during the first baseline phase of the break condition. During these sessions, Chris engaged in problem behavior at an average rate of 6.50 instances per min. Chris continued to engage in problem behavior during the first 4 sessions of the first treatment phase. During these sessions, Chris engaged in problem behavior at an average rate of 5.40 instances per min. During the final three sessions of this condition, however, Chris engaged in no problem behavior. When the break baseline was again implemented, Chris's rates of problem behavior increased to average rate of 2.10 instances per min. When the break treatment was again implemented, Chris engaged in problem behavior at an average rate of 2.32 instances per min. Chris's rate of problem behavior remained variable throughout this second break treatment condition (range, 0.20 to 6.80). Chris's rate of problem behavior initially decreased when the break treatment was implemented however, during sessions 5 and 6 of this phase, Chris's rate of problem behavior increased above levels obtained in the previous baseline condition. Across subsequent treatment sessions, Chris's rate of problem behavior decreased. During the final 3 sessions of this phase, Chris engaged in problem 
behavior at an average rate of 0.4 instances of problem behavior per min. These data indicate that breaks may serve as a reinforcer for Chris's problem behavior.

\section{Discussion}

We assessed if three methods for analyzing descriptive analysis data would produce consistent outcomes. For all participants, identical outcomes were obtained across at least two methods of analysis, but the two methods producing identical results differed across participants (see Table 2 for summary). For Daniel, the methods of analysis described by Martens et al. (2008) and Vollmer et al. (2001) produced identical results. These methods identified attention as a reinforcer for Daniel's problem behavior. For Kameron, the methods of analysis described by Lerman and Iwata (1993) and Martens et al. produced identical results. These methods identified attention, access, and break as reinforcers for Kameron's problem behavior. For Chris, the methods of analysis described by Lerman and Iwata and Vollmer et al. produced identical results. These methods identified attention, access, and break as reinforcers for Chris's problem behavior.

For all participants, attention was identified as a reinforcer for problem behavior by all methods of analysis. These findings replicate previous research showing that descriptive analyses consistently identify attention as a reinforcer for problem behavior (e.g., Pence, Roscoe, Bourret, \& Ahearn, 2009), and that attention is frequently delivered following problem behavior in applied settings (e.g., St. Peter et al., 2005). For the single participant who engaged in consistent problem behavior during the treatment evaluation (Chris), attention was the only reinforcer identified by the descriptive analysis that was not shown to be a reinforcer during intervention. 
The frequency with which attention follows problem behavior in applied settings highlights the importance of conducting functional analyses for identifying the operant function of behavior. The correlation between problem behavior and attention that is frequently identified by descriptive analyses may lead clinicians to assume that attention functions as a reinforcer for problem behavior. This correlation, however, often occurs by chance, due to the high rate at which attention is delivered in applied settings. Most often, problem behavior contacts responseindependent attention, as opposed to response-dependent attention (St. Peter et al., 2005). As such, reliance on descriptive analyses for developing interventions may consistently result in attention-based treatments that are ineffective.

In addition to determining whether the methods of analysis would produce similar results, we assessed whether descriptive analyses would result in effective treatments for reducing problem behavior. For 2 of the 3 participants, Daniel and Kameron, withholding events identified as potential reinforcers by the descriptive analyses did not reliably evoke problem behavior during the intervention evaluation. For Chris, two of the three events identified by the analyses (access and break) did serve as reinforcers for Chris's problem behavior during the intervention evaluation.

It is not clear why descriptive analyses identified reinforcers for Chris but not for Daniel or Kameron. One potential explanation for the lack of correspondence between descriptive analyses and the intervention evaluation is a change in context between the descriptive analysis setting and intervention evaluation setting. In the current experiment, the classroom used for the intervention evaluation was novel for Daniel and Kameron but familiar for Chris. It is possible that Chris's problem behavior had been previously reinforced in that setting, and therefore, stimuli in the classroom became discriminative for problem behavior. As such, results from the 
intervention evaluation differed because discriminative stimuli for problem behavior were present during Chris's intervention evaluation, and were not present during Daniel and Kameron's intervention evaluation. Future research should evaluate if incorporating discriminative stimuli (i.e., the same classroom or teacher) from the descriptive analyses into treatment sessions would result in greater correspondence between the evaluations.

Additionally, for all participants, results from the intervention evaluation may have been affected by having the experimenter serve as therapist during the intervention evaluation sessions. In the present experiment, replacing each child's teacher with a novel experimenter may have removed the most salient discriminative stimulus for problem behavior, thus disrupting problem behavior across the descriptive analysis and intervention evaluation sessions (Ringdahl $\&$ Sellers, 2000).

Previous research has demonstrated that evaluations conducted in the same context are more likely to produce similar results than evaluations conducted in dissimilar contexts (e.g., Anderson, Freeman, \& Scotti, 1999). For example, English and Anderson (2006) compared results from a treatment evaluation conducted in the natural environment to results from a functional analysis conducted in an analog environment and results from a structured descriptive assessment conducted in the natural environment. Treatments based on the results of the structured descriptive assessment were more efficacious than treatments based on the results of the functional analysis. In the current study, our results may have been limited due to the different settings in which the two evaluations were conducted. Conducting the intervention evaluation sessions in the same setting in which descriptive analysis data were collected may have resulted in greater correspondence across the two evaluations. Future research should 
determine whether these methods of analysis result in effective interventions when descriptive analysis observations and intervention sessions are conducted in the same environment.

Results from the present study replicate previous research showing that descriptive analyses often generate multiple hypotheses about the events maintaining problem behavior (e.g., Lalli, Browder, Mace, \& Brown, 1993). In the current study, all methods of analysis generated multiple hypotheses about the events maintaining problem behavior for at least one participant. Additionally, experimental manipulations substantiated results from the descriptive analyses for only one participant (Chris). As such, using descriptive analyses to complete functional behavioral assessments in school settings may result in ineffective or unnecessarily complicated treatments.

It has been suggested that a more viable use of descriptive analyses in school settings may be to assess current behavior-environment relations to determine whether the classroom environment is therapeutic for decreasing a student's problem behavior (e.g., Pence, Roscoe, Bourret, \& Ahearn, 2009). A therapeutic environment would be one in which events identified as reinforcers are more likely to be provided following appropriate behavior than following problem behavior. For example, if attention is a reinforcer for student problem behavior, a therapeutic environment would be one in which attention is more likely to be delivered following appropriate behavior than following problem behavior. It has been suggested that descriptive analyses can be used to determine whether the conditional probability of reinforcers is higher given the occurrence of appropriate behavior than given the occurrence of problem behavior.

Using descriptive analyses for this purpose, however, is impractical in school settings. Analyzing descriptive analysis data according to the three methods of analysis described in the current study requires significant time and training to complete. The method of analysis 
described by Lerman and Iwata (1993) requires a calculation of four conditional probabilities, and the methods of analysis described by Martens et al. (2008) and Vollmer et al. (2001) requires a calculation of two conditional probabilities. In the current study, calculating and graphing these probabilities required at least twice the amount of time necessary to complete descriptive analysis data collection. During a school day, it is unrealistic to expect teachers to have the time to collect these data and calculate the relevant probabilities. Additionally, interpreting the outcomes of these methods of analysis requires significant training. It is unlikely that schools have the time or resources to train teachers to conduct such analyses. As such, using these methods of analyses in school settings is impractical.

Requiring educators to complete FBA's for students who engage in problem behavior necessitates that educators have a method for generating behavioral interventions that is efficient and effective. Although descriptive analyses are common in school settings, findings from the current study indicate that these methods do not consistently identify reinforcers for problem behavior. Additionally, although functional analyses have been demonstrated to be effective for identifying reinforcers for problem behavior, these assessments are rarely implemented by individuals working in nonlaboratory settings (Desrochers, Hile, \& Williams-Moseley, 1997). Future research should focus on the development of alternative methods for generating functionbased interventions that are effective and have strong ecological validity. 


\section{References}

Anderson, C.M., Freeman, K.A., \& Scotti, J.R. (1999). Evaluation of the generalizability (reliability and validity) of analog functional assessment methodology. Behavior Therapy, 30, 31-50.

Carr, J.E., Taylor, C.C., Wallander, R.J., \& Reiss, M.L. (1996). A functional-analytic approach to the diagnosis of a transient tic disorder. Journal of Behavior Therapy and Experimental Psychiatry, 27, 291-297.

Desrochers, M.N., Hile, M.G., \& Williams-Moseley, T.L. (1997). Survey of functional assessment procedures used with individuals who display mental retardation and severe problem behaviors. American Journal of Mental Retardation, 101, 535-546.

Dunlop, G., Kern, L., DePerczel, M., Clarke, S. C., Wilson, D., Childs, K. E., White, R., \& Falk, G. D. (1993). Functional analysis of classroom variables for students with emotional and behavioral disorders. Behavioral Disorders, 18, 275-291.

Durand, V.M., \& Crimmins, D.B. (1987). Assessment and treatment of psychotic speech in an autistic child. Journal of Autism and Developmental Disorders, 17, 1728.

English, C.L., \& Anderson, C.M., (2006). Evaluation of the treatment utility of the analog functional analysis and the structured descriptive assessment. Journal of Positive Behavior Interventions, 8, 212-229.

Fisher, W.W., Lindauer, S.E., Alterson, C.J., \& Thompson, R.H. (1998). Assessment and treatment of destructive behavior maintained by stereotypic object manipulation. Journal of Applied Behavior Analysis, 31, 513-527. 
Hall, S.S. (2005). Comparing descriptive, experimental and informant-based assessments of problem behavior. Research in Developmental Disabilities, 26, 514-526.

Hanley, G. P., Iwata, B. A., \& McCord, B. E. (2003). Functional analysis of problem behavior: A review. Journal of Applied Behavior Analysis, 36, 147-185.

Horner, R. H. (1994). Functional assessment: Contributions and future directions. Journal of Applied Behavior Analysis, 27, 401-404.

Kern, L., Hilt, A.M., \& Gresham, F. (2004). An evaluation of the functional behavioral assessment process used with students with or at risk for emotional and behavioral disorders. Education and Treatment of Children, 27, 440-452.

Lalli, J.S., Browder, D.M., \& Mace, F.C. (1993). Teacher use of descriptive analysis data to implement interventions to decrease students' problem behaviors. Journal of Applied Behavior Analysis, 26, 227-238.

Lerman, D.C., \& Iwata, B.A. (1993). Descriptive and experimental analyses of variables maintaining self-injurious behavior. Journal of Applied Behavior Analysis, 26, 293-319.

Mace, F. C. (1994). The significance and future of functional analysis methodologies. Journal of Applied Behavior Analysis, 27, 385-392.

Mace, F.C., \& Lalli, J.S. (1991). Linking descriptive and experimental analyses in the treatment of bizarre speech. Journal of Applied Behavior Analysis, 24, 553-562.

Martens, B.K., DiGennaro, F.D., Reed, D.D., Szczech, F.M., \& Rosenthal, B.D. (2008). Contingency space analysis: an alternative method for identifying contingent relations from observational data. Journal of Applied Behavior Analysis, 41, 6981. 
Martens, B.K., Gertz, L.E., Werder, C., \& Rymanowski, J. (2010). Agreement between descriptive and experimental analyses of behavior under naturalistic test conditions. Journal of Behavioral Education, 19, 205-221.

Michael, J. (1993). Establishing operations. The Behavior Analyst, 16, 191-206.

Miltenberger, R.G., Long, E.S., Rapp, J.T., Lumley, V., \& Elliot, A.J. (1998). Evaluating the function of hair pulling: a preliminary investigation. Behavior Therapy, 29, 211-219.

Pinkston, E.M., Reese, N.M., LeBlanc, J.M., \& Baer, D.M. (1973). Independent control of a preschool child's aggression and peer interaction by contingent teacher attention. Journal of Applied Behavior Analysis, 6, 115-124.

Pence, S.T., Roscoe, E.M., Bourret, J.C., \& Ahearn, W.H. (2009). Relative contributions of three descriptive methods: Implications for behavioral assessment. Journal of Applied Behavior Analysis, 42, 425-446.

Ringdahl, J.E., \& Sellers, J.A., (2000). The effects of different adults as therapists during functional analyses. Journal of Applied Behavior Analysis, 33, 247-250.

St. Peter C.C., Vollmer, T.R., Bourret, J.C., Borrero, C.S., Sloman, K.N., \& Rapp, J.T. (2005). On the role of attention in naturally occurring matching relations. Journal of Applied Behavior Analysis, 38, 429-443.

Sugai, G., Lewis-Palmer, T., \& Hagan-Burke, S. (2000). Overview of the functional behavioral assessment process. Exceptionality, 8, 149-160.

Thompson, R.H., \& Iwata, B.A. (2007). A descriptive analysis of social consequences following problem behavior. Journal of Applied Behavior Analysis, 34, 169-178.

Vollmer, T.R., Borrero, J.C., Wright, C.S., Van Camp, C., \& Lalli, J.S. (2001). Identifying possible contingencies during descriptive analyses of severe behavior 
disorders. Journal of Applied Behavior Analysis, 34, 269-287.

Weber, K.P., Killu, K., Derby, K.M., \& Barretto, A. (2005). The status of functional behavior assessment (FBA): adherence to standard practice in FBA methodology. Psychology in the Schools, 42, 737-734. 
Table 1

Probabilities calculated for each method of analysis

\begin{tabular}{|c|c|c|}
\hline & Method & Probabilities Calculated \\
\hline \multirow[t]{4}{*}{1} & Lerman and Iwata, 1993 & $\frac{\text { Intervals containing behavior that follow an antecedent event }}{\text { Total \# of intervals with behavior }}$ \\
\hline & & $\frac{\text { Intervals containing an antecedent event that precede behavior }}{\text { Total \# of intervals with event }}$ \\
\hline & & $\frac{\text { Intervals containing behavior that precede a consequent event }}{\text { Total \# of intervals with behavior }}$ \\
\hline & & $\frac{\text { Intervals containing a consequent event that follow behavior }}{\text { Total \# of intervals with event }}$ \\
\hline & Vollmer et al., 2001 & $\frac{\# \text { of instances of behavior followed by subsequent event }}{\text { Total \# of instances of behavior }}$ \\
\hline & & $\frac{\# \text { of seconds with subsequent event }}{\text { Total \# of seconds }}$ \\
\hline & Martens et al., 2008 & $\frac{\text { Intervals containing behavior and subsequent event }}{\text { Total \# of behavior }}$ \\
\hline & & Intervals containing subsequent event \\
\hline & & Total \# of intervals without behavior \\
\hline
\end{tabular}


Table 2

A Comparison of the Putative Reinforcers Identified Across the Three Methods of Analysis

Putative Reinforcers Identified by Descriptive Analyses

\begin{tabular}{llll}
\hline $\begin{array}{l}\text { Subject } \\
(2001)\end{array}$ & \multicolumn{1}{c}{ Lerman \& Iwata (1993) } & Martens et al. (2008) & Vollmer et al. \\
\hline \multirow{2}{*}{ Daniel } & $\begin{array}{l}\text { Attention } \\
\text { Access } \\
\text { Break }\end{array}$ & Attention & Attention \\
& Attention & & \\
Kameron & Access & Attention & Attention \\
& Break & Access & \\
& Bttention & Break & \\
& Access & Attention & Attention \\
Chris & Break & & Access \\
& & & Break \\
\hline
\end{tabular}



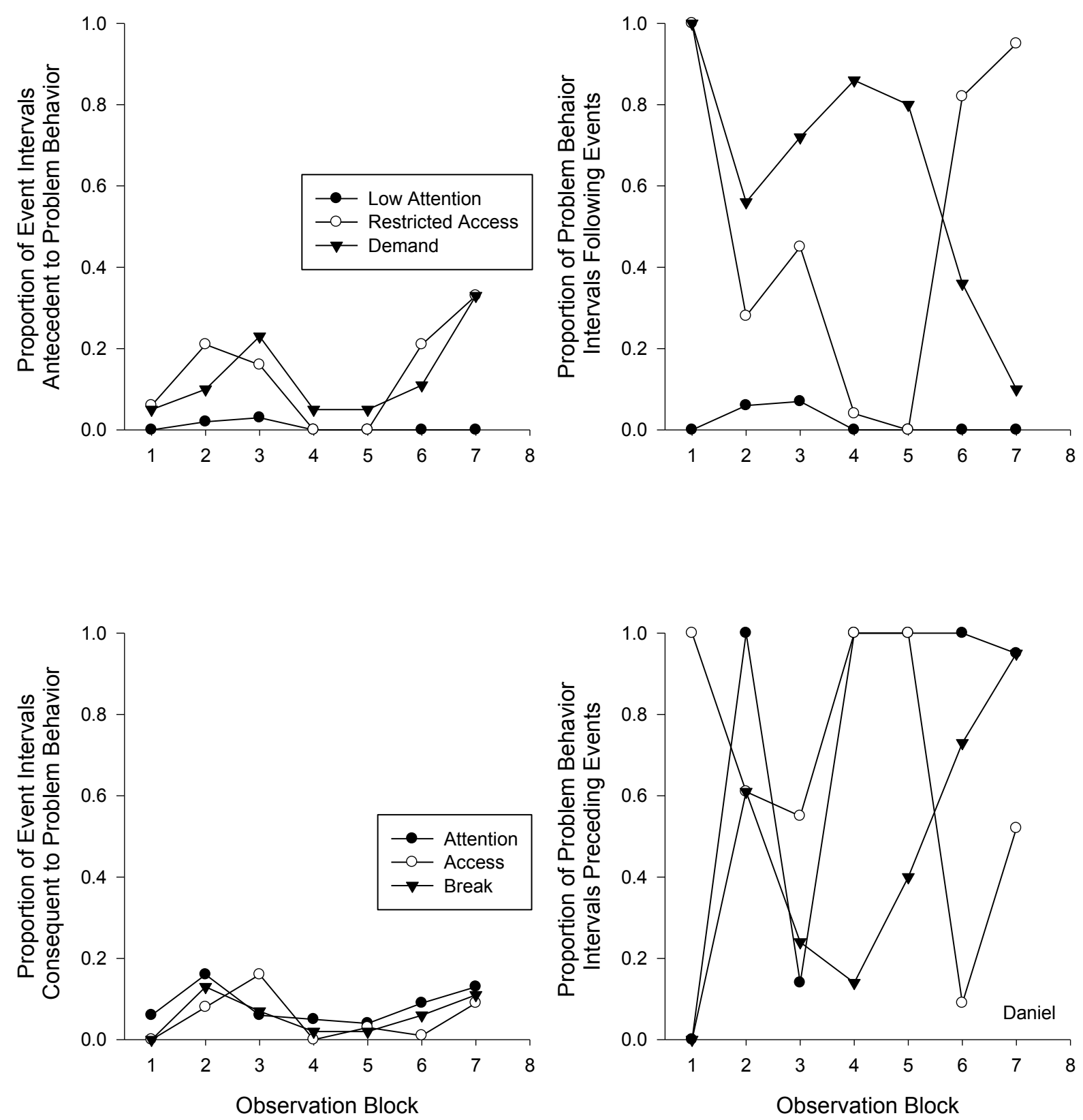

Figure 1. Graph of Daniel's descriptive analysis data analyzed according to the method described by Lerman and Iwata (1993). 

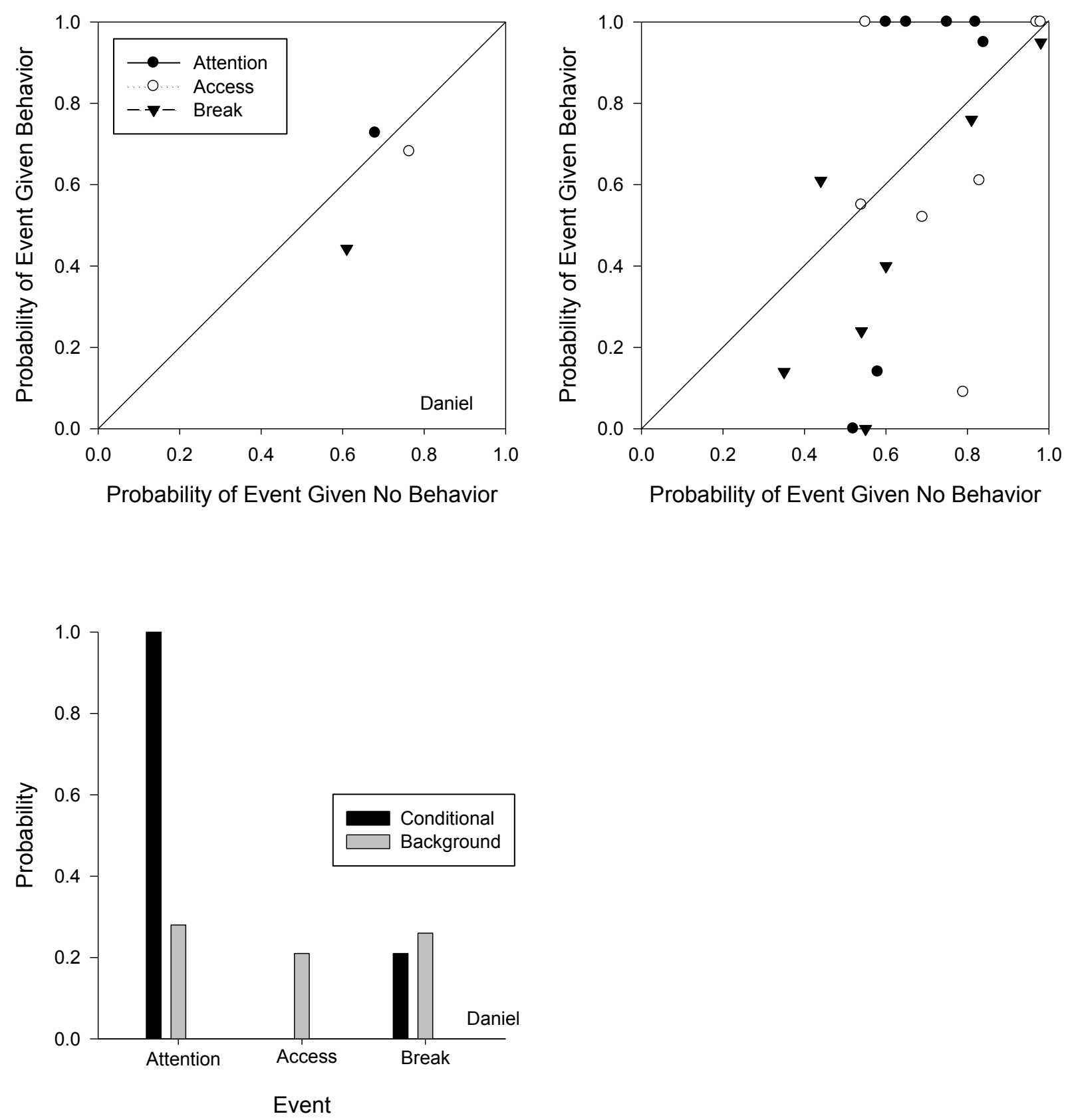

Figure 2. Graph of Daniel's descriptive analysis data analyzed according to the methods of analysis described by Martens et al. (2008) and Vollmer et al. (2001). The top panel represents Daniel's data analyzed according to the method described by Martens et al. and the bottom panel represents Daniel's data analyzed according to the method described by Vollmer et al. 

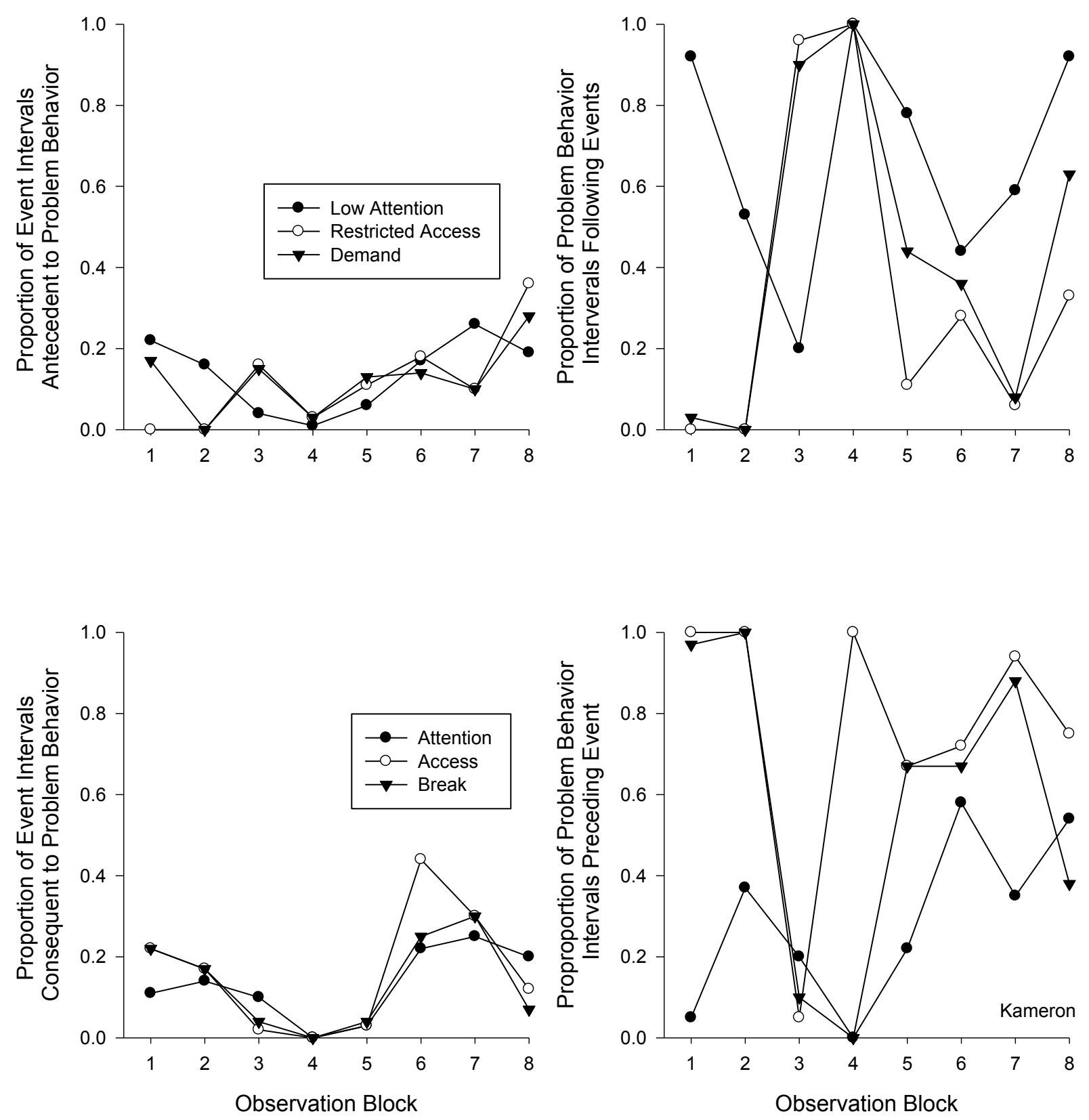

Figure 3. Graph of Kameron's descriptive analysis data analyzed according to the method described by Lerman and Iwata (1993). 


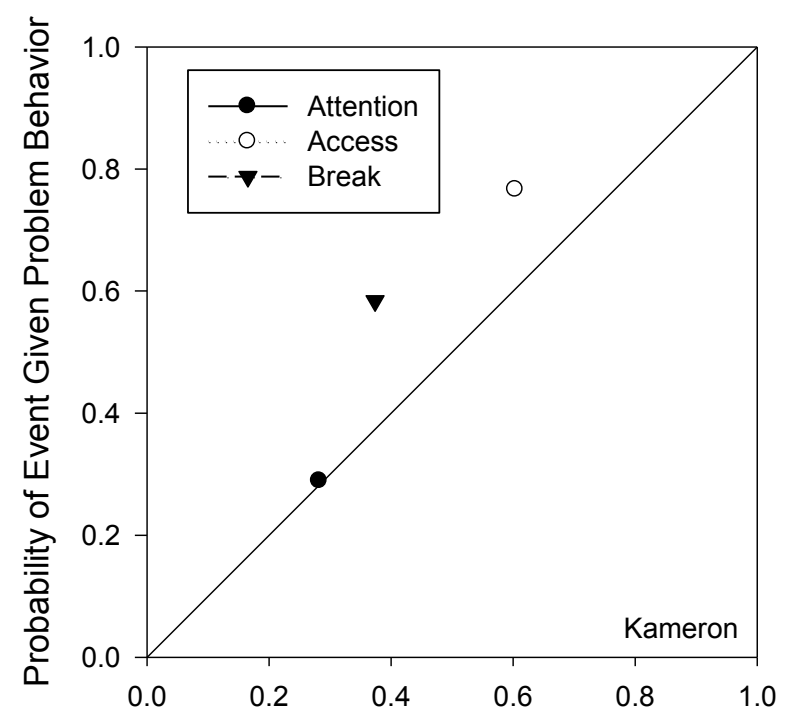

Probability of Event Given No Problem Behavior

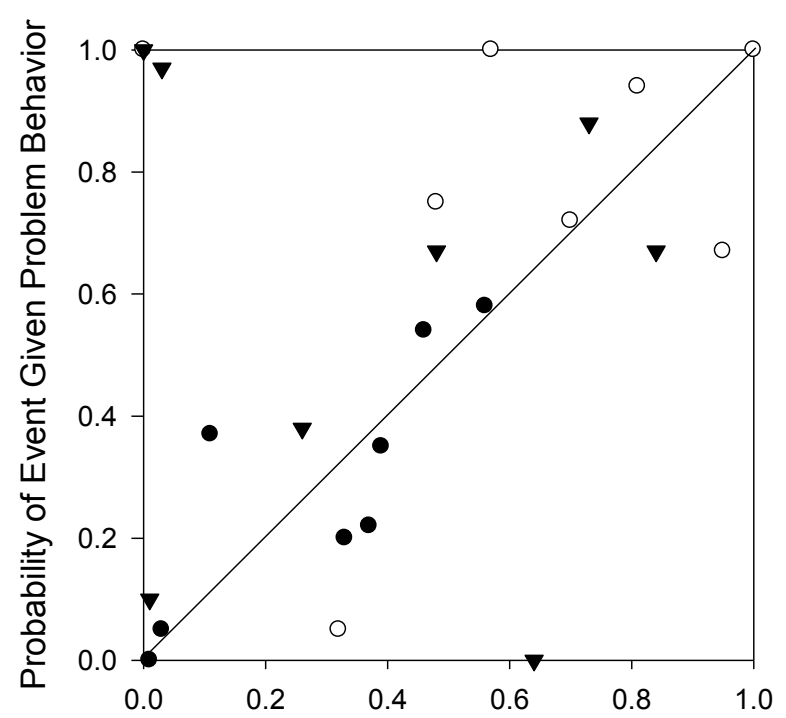

Probability of Event Given No Problem Behavior

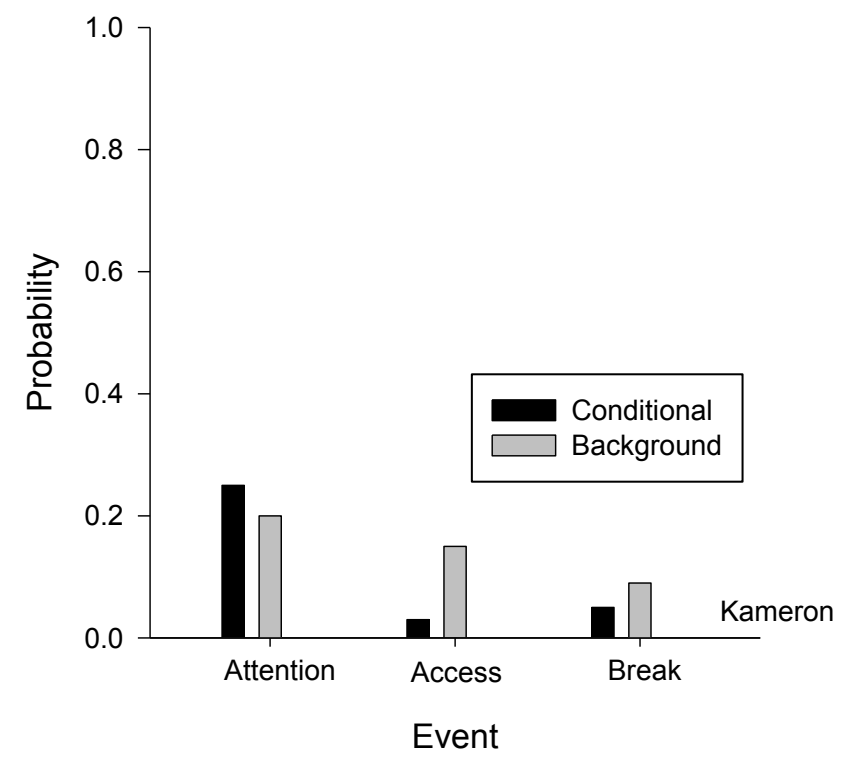

Figure 4. Graph of Kameron's descriptive analysis data analyzed according to the methods described by Martens et al. (2008) and Vollmer et al. (2001). The top panel represents Kameron's descriptive analysis data analyzed according to the method described by Martens et al and the bottom panel represents Kameron's data analyzed according to the method described by Vollmer et al. 

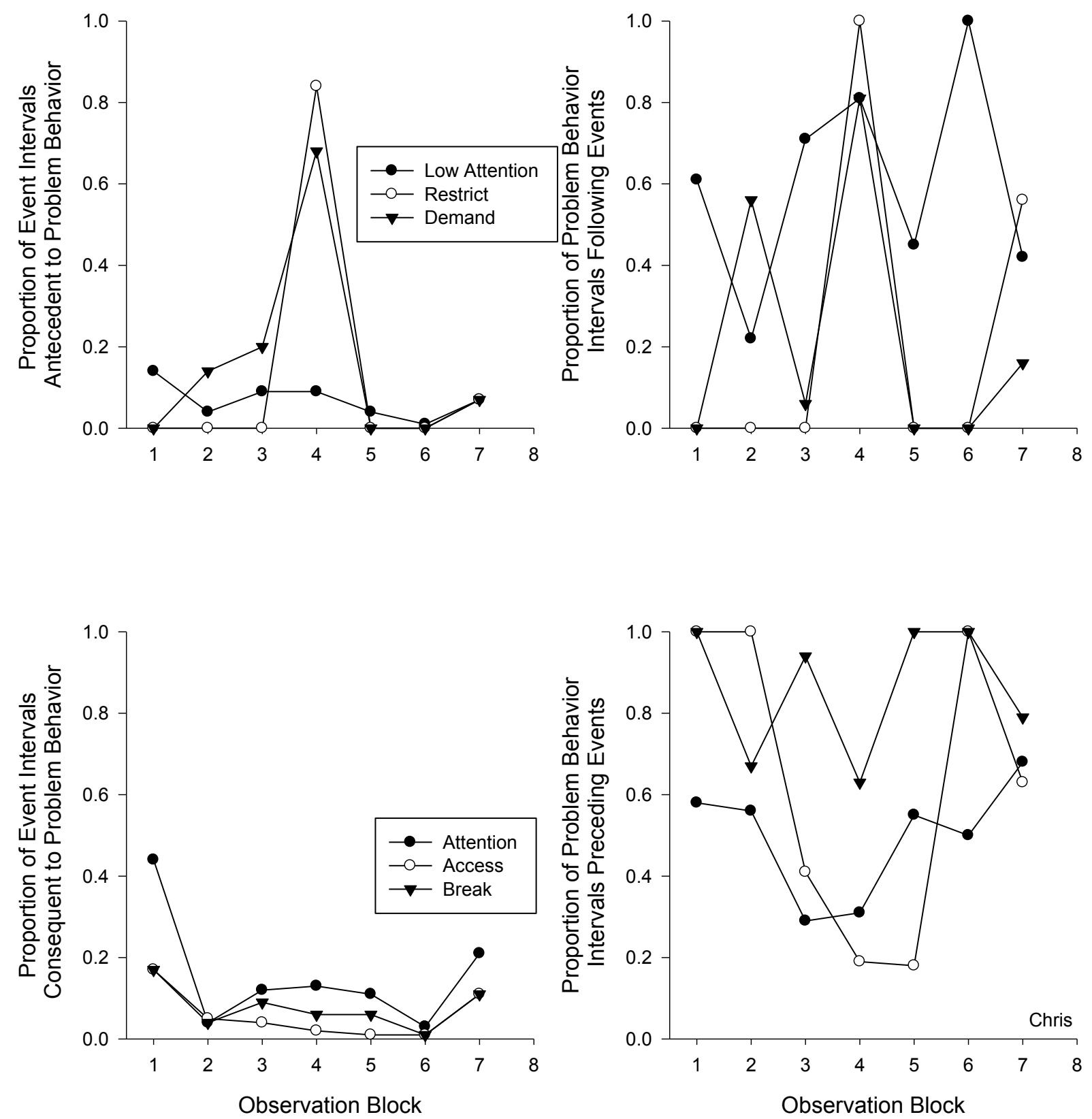

Figure 5. Graph of Chris's descriptive analysis data analyzed according to the method described by Lerman and Iwata (1993). 


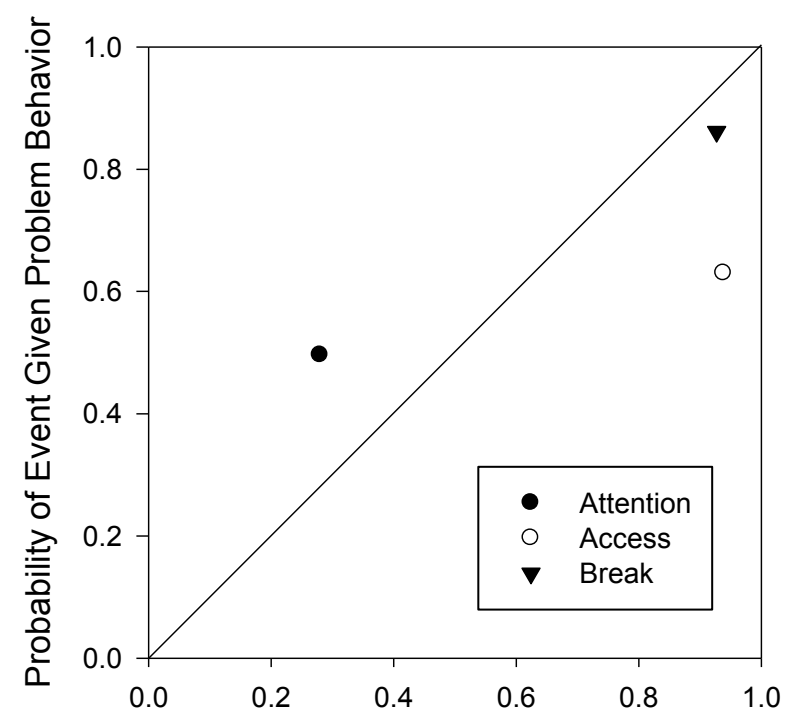

Probability of Event Given No Problem Behavior

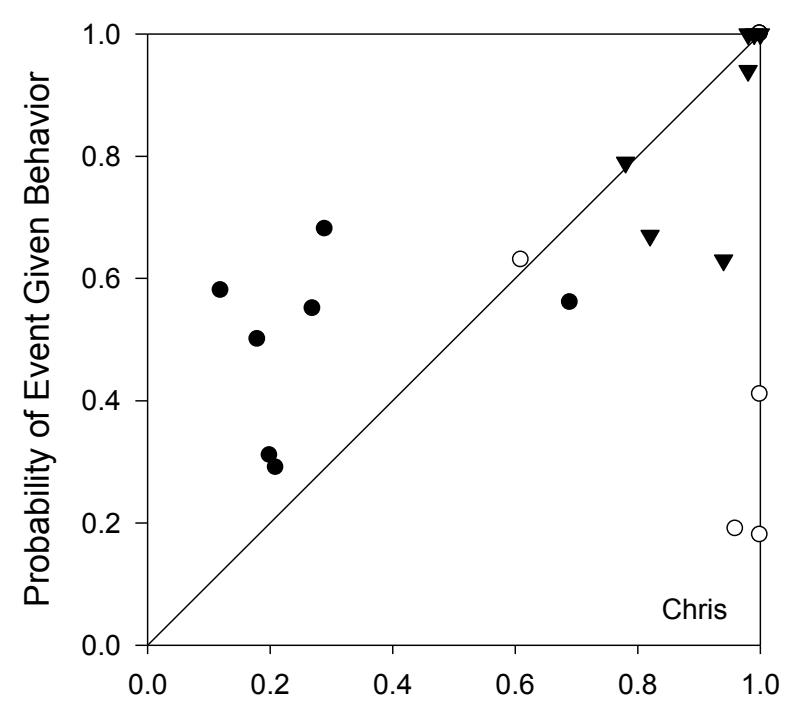

Probability of Event Given No Behavior

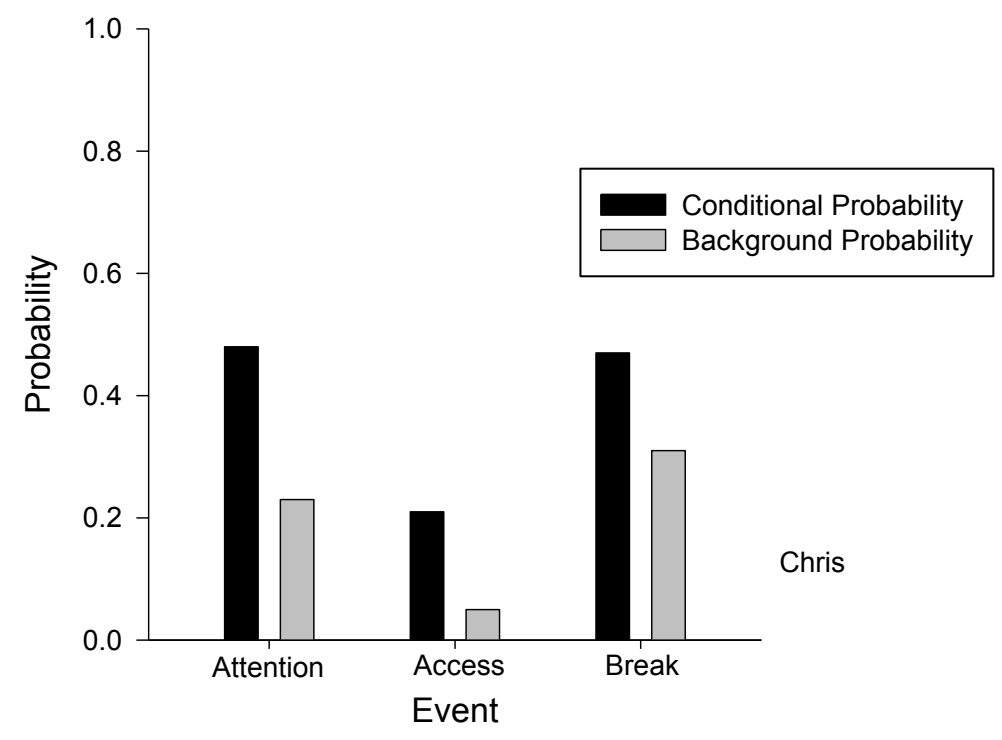

Figure 6. Graph of Chris's descriptive analysis data analyzed according to the methods described by Martens et al. (2008) and Vollmer et al. (2001). The top panel represents Chris's data analyzed according to the method described by Martens et al. and the bottom panel represents Chris's data analyzed according to the method described by Vollmer et al. 


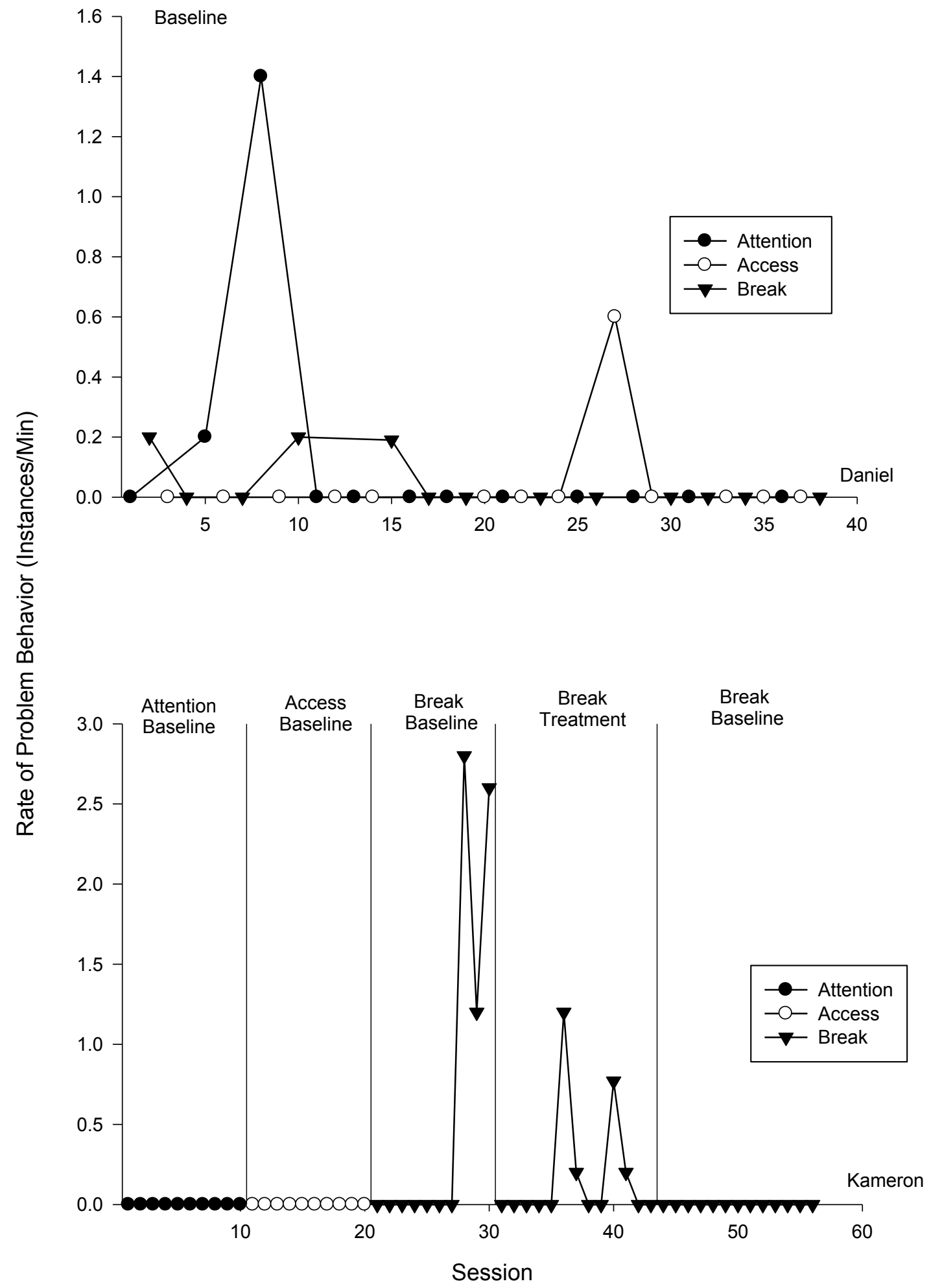

Figure 7. Graph of treatment components for all participants. The top panel displays treatment results for Daniel and the middle panel displays treatment results for Kameron. 


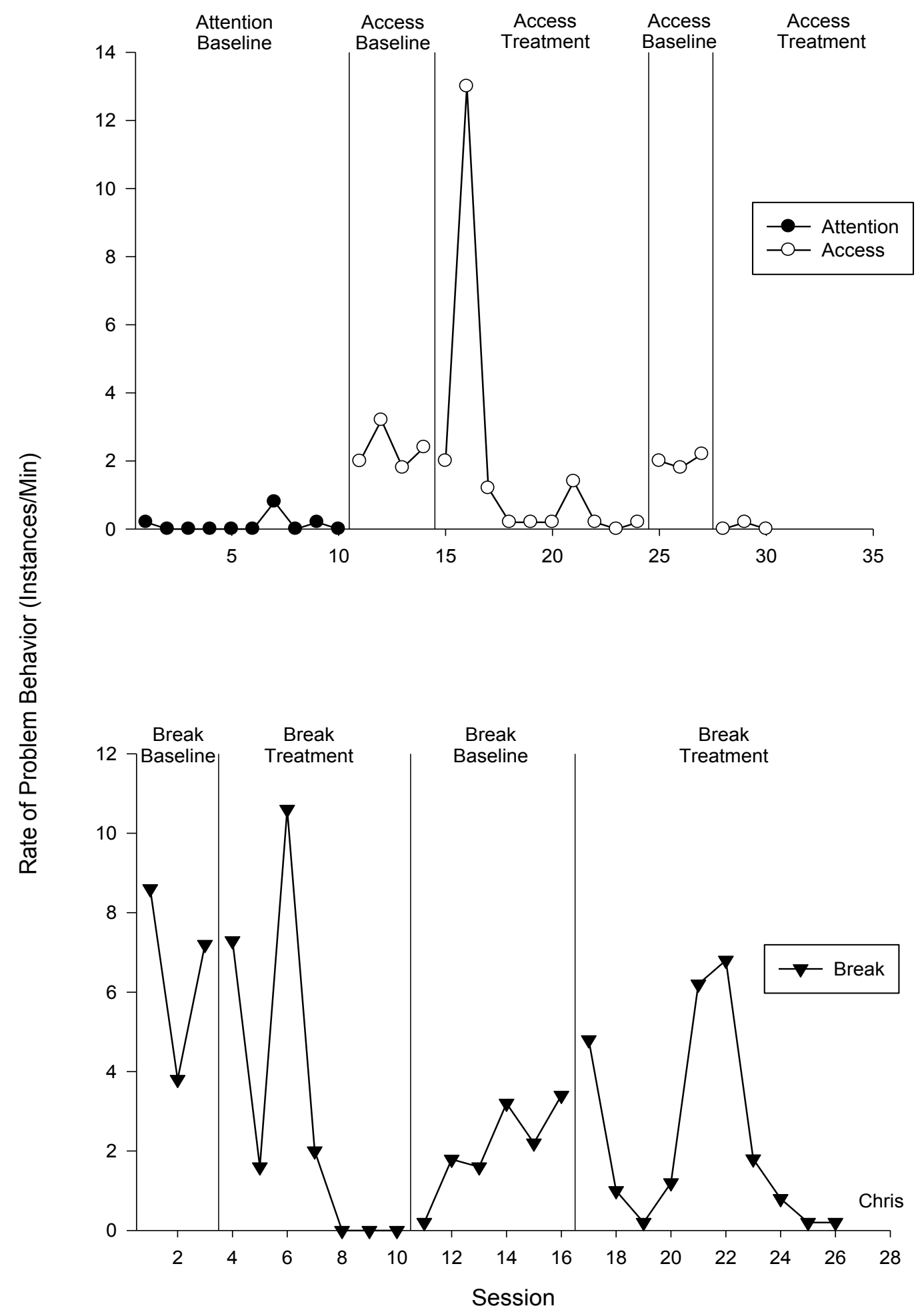

Figure 8. Graph of treatment results for Chris. The top panel displays treatment results from the attention and access intervention and the bottom panel displays treatment results from the break intervention. 